\title{
SUNYAEV-ZEL'DOVICH-MEASURED PRESSURE PROFILES FROM THE BOLOCAM X-RAY/SZ GALAXY CLUSTER SAMPLE
}

\author{
J. Sayers ${ }^{1}$, N. G. Czakon ${ }^{1}$, A. Mantz ${ }^{2}$, S. R. Golwala ${ }^{1}$, S. Ameglio ${ }^{3}$, T. P. Downes ${ }^{1}$, P. M. Koch ${ }^{4}$, K.-Y. Lin ${ }^{4}$, \\ B. J. Maughan ${ }^{5}$, S. M. Molnar ${ }^{6}$, L. Moustakas ${ }^{7}$, T. Mroczkowski ${ }^{1,7,8}$, E. Pierpaoli ${ }^{3}$, J. A. Shitanishi ${ }^{3}$, \\ S. Siegel ${ }^{1}$, K. UMETSU ${ }^{4}$, AND N. VAN DER PYL ${ }^{5}$ \\ ${ }^{1}$ Division of Physics, Math, and Astronomy, California Institute of Technology, Pasadena, CA 91125, USA; jack@ caltech.edu \\ ${ }^{2}$ Kavli Institute for Cosmological Physics, University of Chicago, 5640 South Ellis Avenue, Chicago, IL 60637, USA \\ ${ }^{3}$ University of Southern California, Los Angeles, CA 90089, USA \\ ${ }^{4}$ Institute of Astronomy and Astrophysics, Academia Sinica, P.O. Box 23-141, Taipei 10617, Taiwan \\ ${ }^{5}$ H. H. Wills Physics Laboratory, University of Bristol, Tyndall Avenue, Bristol Bs8 ITL, UK \\ ${ }^{6}$ LeCosPA Center, National Taiwan University, Taipei 10617, Taiwan \\ 7 Jet Propulsion Laboratory, Pasadena, CA 91109, USA \\ Received 2012 November 5; accepted 2013 March 19; published 2013 April 26
}

\begin{abstract}
We describe Sunyaev-Zel'dovich (SZ) effect measurements and analysis of the intracluster medium (ICM) pressure profiles of a set of 45 massive galaxy clusters imaged using Bolocam at the Caltech Submillimeter Observatory. We deproject the average pressure profile of our sample into 13 logarithmically spaced radial bins between $0.07 R_{500}$ and $3.5 R_{500}$, and we find that a generalized Navarro, Frenk, and White (gNFW) profile describes our data with sufficient goodness-of-fit and best-fit parameters $\left(C_{500}, \alpha, \beta, \gamma, P_{0}=1.18,0.86,3.67,0.67,4.29\right)$. We use X-ray data to define cool-core and disturbed subsamples of clusters, and we constrain the average pressure profiles of each of these subsamples. We find that, given the precision of our data, the average pressure profiles of disturbed and cool-core clusters are consistent with one another at $R \gtrsim 0.15 R_{500}$, with cool-core systems showing indications of higher pressure at $R \lesssim 0.15 R_{500}$. In addition, for the first time, we place simultaneous constraints on the mass scaling of cluster pressure profiles, their ensemble mean profile, and their radius-dependent intrinsic scatter between $0.1 R_{500}$ and $2.0 R_{500}$. The scatter among profiles is minimized at radii between $\simeq 0.2 R_{500}$ and $\simeq 0.5 R_{500}$, with a value of $\simeq 20 \%$. These results for the intrinsic scatter are largely consistent with previous analyses, most of which have relied heavily on X-ray derived pressures of clusters at significantly lower masses and redshifts compared to our sample. Therefore, our data provide further evidence that cluster pressure profiles are largely universal with scatter of $\simeq 20 \%-40 \%$ about the universal profile over a wide range of masses and redshifts.
\end{abstract}

Key words: galaxies: clusters: general - galaxies: clusters: intracluster medium

Online-only material: color figures

\section{INTRODUCTION}

Massive galaxy clusters, the largest virialized systems in the universe, appear to be exceptionally regular objects. This is especially true at intermediate radii outside of the core (where complicated baryonic physics plays a large role), and inside the actively accreting outer regions (where non-equilibrium effects become significant). In these intermediate regions simple, selfsimilar scalings based on hydrostatic equilibrium and gravitational physics describe observations and simulations quite well (Kaiser 1986; Kravtsov \& Borgani 2012). Specifically, a range of observational results show approximately universal behavior among mass and intracluster medium (ICM) profiles after scaling by characteristic overdensity radii such as $R_{500}{ }^{9}$ and by selfsimilar mass- and redshift-dependent normalizations (Vikhlinin et al. 2006; Pratt et al. 2007; Nagai et al. 2007; Cavagnolo et al. 2009; Arnaud et al. 2010, hereafter A10; Planck Collaboration 2013, hereafter P12; Navarro et al. 1996; Umetsu et al. 2011; Kravtsov \& Borgani 2012; Walker et al. 2012). For example, the cluster-to-cluster dispersion (intrinsic scatter) observed in these

\footnotetext{
8 NASA Einstein Postdoctoral Fellow.

$9 \quad R_{500}$ denotes the radius where the average enclosed mass density is 500 times the critical density. Throughout this manuscript we define quantities at, or enclosed within, this characteristic radius.
}

scaled profiles outside of the core regions and inside $R_{500}$ is generally $\simeq 10 \%-40 \%$ for entropy (Pratt et al. 2006; Cavagnolo et al. 2009; Pratt et al. 2010; Walker et al. 2012), gas density (Vikhlinin et al. 2006; Croston et al. 2008; Maughan et al. 2012; Eckert et al. 2012), temperature (Vikhlinin et al. 2006; Pratt et al. 2007; Leccardi \& Molendi 2008), and pressure (A10; Sun et al. 2011). In particular, both simulations and observations indicate low cluster-to-cluster dispersion in pressure profiles at intermediate radii (Borgani et al. 2004; Nagai et al. 2007; Piffaretti \& Valdarnini 2008; A10; Plagge et al. 2010; Bonamente et al. 2012; P12).

Historically, observational studies of the ICM pressure have relied almost exclusively on X-ray data. These data have provided precise constraints on the pressure profiles in the inner regions of clusters $\left(R \leqslant R_{500}\right)$, but the density-squared dependence of the X-ray surface brightness makes it difficult to study the ICM at large radii with X-rays. Although several $\mathrm{X}$-ray results have extended beyond $R_{500}$ in individual clusters or small sets of clusters (George et al. 2009; Bautz et al. 2009; Reiprich et al. 2009; Simionescu et al. 2011; Walker et al. 2012), with current X-ray instrumentation it is infeasible to extend such studies to large samples of clusters. Consequently, two groups have used a hybrid approach with X-ray data at small radii $\left(R \lesssim R_{500}\right)$ and simulations at large radii $\left(R \gtrsim R_{500}\right)$ in order to constrain the average pressure profile at all relevant radial scales 
(Nagai et al. 2007; A10). These X-ray and simulation-based results imply that cluster pressure profiles are approximately universal over a wide range of masses and radial scales, with low intrinsic scatter that is minimized near $0.5 R_{500}$ at $\lesssim 20 \%$ (e.g., A10; Nagai et al. 2007; Sun et al. 2011).

The Sunyaev-Zel'dovich (SZ) effect signal (Sunyaev \& Zel'dovich 1972), which is proportional to the density of the ICM and therefore falls more slowly with radius compared with the X-ray brightness, can be exploited to study the ICM pressure at large radii. Although some initial studies using Wilkinson Microwave Anisotropy Probe (WMAP) SZ data showed large inconsistencies with the established X-ray results (Lieu et al. 2006; Bielby \& Shanks 2007), recent results have shown that SZ data from WMAP, Planck, and ground-based receivers provide a picture of the ICM that is consistent with X-ray measurements at current observational precision (Plagge et al. 2010; Melin et al. 2011; Planck Collaboration 2011; Komatsu et al. 2011; Bonamente et al. 2012). In particular, the South Pole Telescope (SPT) was able to measure the average SZ pressure profile out to $\simeq 2 R_{500}$ for a sample of 15 clusters (Plagge et al. 2010), finding results that were similar to previous X-ray/simulation results at small/large radii (e.g., the sample of 31 REXCESS clusters studied by A10). Recently, a combination of XMM-Newton $\mathrm{X}$-ray data at small radii and Planck SZ effect data at large radii was used to constrain the average pressure profile out to $3 R_{500}$ for a sample of 62 Planck-selected clusters (P12). These results were again largely consistent with previous analyses, and the $\mathrm{X}$-ray and SZ data agreed quite well in the overlapping region at intermediate radii. Altogether, X-ray and SZ data, along with simulations, are converging to a uniform picture of the average cluster pressure profile over a wide range of angular scales.

This manuscript is arranged as follows. In Section 2 we describe our sample of 45 massive clusters, and in Section 3 we provide the details of our SZ and X-ray data reduction. We then present our method for deprojecting pressure profiles from our spatially-filtered SZ images in Section 4. In Section 5.1, we describe parametric fits to these deprojected profiles, and compare the results of our fits to the results from a range of previous analyses. Then, in Section 5.2, we use a Gaussian process formalism to simultaneously constrain the pressureprofile mass scaling, the ensemble mean profile, and the radiusdependent intrinsic scatter about this mean profile. Finally, we provide a summary of our results in Section 6.

\section{CLUSTER SAMPLE}

Between 2006 November and 2012 March we used Bolocam to image the SZ signals from a sample of 45 clusters that have Chandra X-ray exposures (hereafter the Bolocam X-ray/SZ or BOXSZ sample). The Bolocam SZ observations and some general properties of the BOXSZ sample are summarized in Table 1, and the details of the Chandra X-ray data and analysis are given in Tables 2 and 3. The BOXSZ sample contains two previously defined subsamples, the 25 object Cluster Lensing and Supernova Survey with Hubble (CLASH) sample (Postman et al. 2012) and the 12 cluster MACS high- $z$ sample (Ebeling et al. 2007). The remaining clusters were selected in an ad hoc manner, with a general emphasis on massive and/or high redshift systems. The clusters span the redshift range $0.15 \leqslant$ $z \leqslant 0.89$, with a median redshift of 0.42 and more than $60 \%$ of the sample lying between $0.35 \leqslant z \leqslant 0.59$. The clusters are among the most massive known, with a median X-ray derived mass of $M_{500}=9 \times 10^{14} M_{\odot}$ assuming a constant gas mass fraction (we computed these masses using a reference $h=0.7$,
$\Omega_{m}=0.3$ flat $\Lambda \mathrm{CDM}$ cosmology, and this same cosmology was used to calculate all other physical quantities presented in this manuscript). The clusters in the BOXSZ sample span a range of dynamical states, from relaxed systems with well defined cool cores like A1835 (Peterson et al. 2001; Schmidt et al. 2001) to clusters undergoing major merger events like MACS J0717.5 (Ebeling et al. 2001; Edge et al. 2003; Mroczkowski et al. 2012).

Based on previous results that show the projected X-ray luminosity ratio is an accurate indicator of cool-core clusters (Mantz 2009; Böhringer et al. 2010), we define a cool-core subsample of the BOXSZ sample as those clusters with a projected X-ray luminosity ratio

$$
L_{\text {rat }}=\frac{L\left(R<0.05 R_{500}\right)}{L\left(R<R_{500}\right)} \geqslant 0.17 .
$$

According to this definition, 17/45 of the BOXSZ clusters are cool-core systems (see Table 3 ). We note that these cool-core systems are in general at the low redshift end of our sample, with a median redshift of $z=0.36$. We speculate that this result is due at least in part to the fact that the cool-core fraction of clusters drops with increasing redshift (e.g., Vikhlinin et al. 2007; Santos et al. 2010), although selection effects may also play a role due to the ad hoc manner in which the BOXSZ sample was chosen.

We define a disturbed subsample of the BOXSZ sample as those clusters with an X-ray centroid shift parameter of $w_{500} \geqslant 0.01$ (see Section 3.2 for a full description of how we compute $\left.w_{500}\right)$. The centroid shift parameter is widely used to classify disturbed systems (e.g., Maughan et al. 2008; Pratt et al. 2009; Maughan et al. 2012), and we adopt the same threshold $\left(w_{500} \geqslant 0.01\right)$ as Pratt et al. (2009). Based on this criteria, 16/45 clusters in the BOXSZ sample are disturbed, and the disturbed systems are generally at the high redshift end of the full sample with a median redshift of $z=0.52$. This result is not surprising given that the cool-core systems are generally at low redshift and cool cores are a good indicator that the cluster is not disturbed (only 2/45 clusters in the BOXSZ sample have both a cool core and are disturbed).

This redshift asymmetry between the cool-core and disturbed subsamples within the BOXSZ sample has an impact on our analysis of the average pressure profile of the sample. As we describe in Section 4, we scale the radial coordinate of each cluster by $R_{500}$, since the shape of the pressure profiles is expected to be self-similar after scaling by this radius. However, the angular dynamic range of our data is limited by Bolocam's 58 arcsec full-width at half-maximum (FWHM) point-spread function (PSF) and by the 14 arcmin size of the Bolocam images (i.e., our SZ data are sensitive to a minimum radius of $\simeq 29 \operatorname{arcsec}$ and a maximum radius of $\simeq 10 \mathrm{arcmin}$ ). Since the value of $R_{500}$ in physical units is fairly constant over our sample $(\simeq 1.5 \mathrm{Mpc})$, this means that the value of $R_{500}$ varies significantly in angular size over the redshift range of our sample, from 2 arcmin to 9 arcmin. This can be clearly seen in Figure 1, where we show the Bolocam integration time as a function of scaled radius for the BOXSZ sample. On average, we obtained longer integrations for the preferentially high redshift disturbed clusters, and this compensates for their smaller angular size compared with the preferentially low redshift cool-core clusters to produce nearly equal integration times at $R \lesssim R_{500}$. However, the increased integration time and smaller angular sizes of the disturbed clusters results in significantly more integration time outside $R_{500}$ compared with the cool-core clusters. Consequently, the average SZ pressure profile of the BOXSZ sample is constrained 
Table 1

Bolocam X-Ray/SZ (BOXSZ) Cluster Sample

\begin{tabular}{|c|c|c|c|c|c|c|}
\hline Cluster & Redshift & $\begin{array}{l}\text { R.A. } \\
(\mathrm{J} 2000)\end{array}$ & $\begin{array}{l}\text { Decl. } \\
\text { (J2000) }\end{array}$ & $\begin{array}{l}\text { Obs. Time } \\
\text { (hours) }\end{array}$ & $\begin{array}{c}\text { Noise } \\
\left(\mu \mathrm{K}_{\mathrm{CMB}} \text {-amin }\right)\end{array}$ & Peak S/N \\
\hline A2204 & $0.151^{\mathrm{a}}$ & $16: 32: 47.2$ & $+05: 34: 33$ & 12.7 & 18.5 & 22.3 \\
\hline A383 & $0.188^{\mathrm{b}}$ & $02: 48: 03.3$ & $-03: 31: 46$ & 24.3 & 18.9 & 9.6 \\
\hline A209 & $0.206^{\mathrm{a}}$ & 01:31:53.1 & $-13: 36: 48$ & 17.8 & 22.3 & 13.9 \\
\hline A963 & $0.206^{\mathrm{a}}$ & 10:17:03.6 & $+39: 02: 52$ & 11.0 & 35.7 & 8.3 \\
\hline A1423 & $0.213^{\mathrm{a}}$ & 11:57:17.4 & $+33: 36: 40$ & 11.5 & 31.7 & 5.8 \\
\hline A2261 & $0.224^{\mathrm{a}}$ & $17: 22: 27.0$ & $+32: 07: 58$ & 17.5 & 15.9 & 10.2 \\
\hline A2219 & $0.228^{\mathrm{a}}$ & $16: 40: 20.3$ & $+46: 42: 30$ & 6.3 & 39.6 & 11.1 \\
\hline A267 & $0.230^{\mathrm{a}}$ & $01: 52: 42.2$ & $+01: 00: 30$ & 20.7 & 23.0 & 9.6 \\
\hline RX J2129.6 & $0.235^{\mathrm{a}}$ & $21: 29: 39.7$ & $+00: 05: 18$ & 16.0 & 23.7 & 8.0 \\
\hline A1835 & $0.253^{\mathrm{a}}$ & 14:01:01.9 & $+02: 52: 40$ & 14.0 & 16.2 & 15.7 \\
\hline A697 & $0.282^{\mathrm{a}}$ & $08: 42: 57.6$ & $+36: 21: 57$ & 14.3 & 17.4 & 22.6 \\
\hline A611 & $0.288^{\mathrm{b}}$ & 08:00:56.8 & $+36: 03: 26$ & 18.7 & 25.0 & 10.8 \\
\hline MS 2137 & $0.313^{\mathrm{b}}$ & $21: 40: 15.1$ & $-23: 39: 40$ & 12.8 & 27.3 & 6.5 \\
\hline AS1063 & $0.348^{\mathrm{c}}$ & $22: 48: 44.8$ & $-44: 31: 45$ & 5.5 & 48.6 & 10.2 \\
\hline MACS J1931.8 & $0.352^{\mathrm{a}}$ & $19: 31: 49.6$ & $-26: 34: 34$ & 7.5 & 28.7 & 10.1 \\
\hline MACS J1115.8 & $0.355^{\mathrm{a}}$ & $11: 15: 51.9$ & $+01: 29: 55$ & 15.7 & 22.8 & 10.9 \\
\hline MACS J1532.9 & $0.363^{\mathrm{a}}$ & $15: 32: 53.8$ & $+30: 20: 59$ & 14.8 & 22.3 & 8.0 \\
\hline A370 & $0.375^{\mathrm{d}}$ & 02:39:53.2 & $-01: 34: 38$ & 11.8 & 28.9 & 12.8 \\
\hline MACS J1720.3 & $0.387^{\mathrm{a}}$ & $17: 20: 16.7$ & $+35: 36: 23$ & 16.8 & 23.5 & 10.6 \\
\hline ZWCL 0024 & $0.395^{\mathrm{e}}$ & $00: 26: 35.8$ & $+17: 09: 41$ & 8.3 & 26.6 & 3.3 \\
\hline MACS J2211.7 & $0.396^{\mathrm{a}}$ & $22: 11: 45.9$ & $-03: 49: 42$ & 6.5 & 38.6 & 14.7 \\
\hline MACS J0429.6 & $0.399^{\mathrm{a}}$ & $04: 29: 36.0$ & $-02: 53: 06$ & 17.0 & 24.1 & 8.9 \\
\hline MACS J0416.1 & $0.420^{\mathrm{f}}$ & 04:16:08.8 & $-24: 04: 14$ & 7.8 & 29.3 & 8.5 \\
\hline MACS J0451.9 & $0.430^{\mathrm{c}}$ & $04: 51: 54.7$ & $+00: 06: 19$ & 14.2 & 22.7 & 8.1 \\
\hline MACS J1206.2 & $0.439^{\mathrm{a}}$ & $12: 06: 12.3$ & $-08: 48: 06$ & 11.3 & 24.9 & 21.7 \\
\hline MACS J0417.5 & $0.443^{\mathrm{a}}$ & $04: 17: 34.3$ & $-11: 54: 27$ & 9.8 & 22.7 & 22.7 \\
\hline MACS J0329.6 & $0.450^{\mathrm{b}}$ & 03:29:41.5 & $-02: 11: 46$ & 10.3 & 22.5 & 12.1 \\
\hline MACS J1347.5 & $0.451^{\mathrm{a}}$ & $13: 47: 30.8$ & $-11: 45: 09$ & 15.5 & 19.7 & 36.6 \\
\hline MACS J1311.0 & $0.494^{\mathrm{b}}$ & $13: 11: 01.7$ & $-03: 10: 40$ & 14.2 & 22.5 & 9.6 \\
\hline MACS J2214.9 & $0.503^{\mathrm{a}}$ & $22: 14: 57.3$ & $-14: 00: 11$ & 7.2 & 27.3 & 12.6 \\
\hline MACS J0257.1 & $0.505^{\mathrm{a}}$ & 02:57:09.1 & $-23: 26: 04$ & 5.0 & 39.0 & 10.1 \\
\hline MACS J0911.2 & $0.505^{\mathrm{a}}$ & 09:11:10.9 & $+17: 46: 31$ & 6.2 & 33.5 & 4.8 \\
\hline MACS J0454.1 & $0.538^{\mathrm{a}}$ & 04:54:11.4 & $-03: 00: 51$ & 14.5 & 18.2 & 24.3 \\
\hline MACS J1423.8 & $0.543^{\mathrm{a}}$ & $14: 23: 47.9$ & $+24: 04: 43$ & 21.7 & 22.3 & 9.4 \\
\hline MACS J1149.5 & $0.544^{\mathrm{a}}$ & $11: 49: 35.4$ & $+22: 24: 04$ & 17.7 & 24.0 & 17.4 \\
\hline MACS J0018.5 & $0.546^{\mathrm{a}}$ & $00: 18: 33.4$ & $+16: 26: 13$ & 9.8 & 21.0 & 15.7 \\
\hline MACS J0717.5 & $0.546^{\mathrm{a}}$ & $07: 17: 32.1$ & $+37: 45: 21$ & 12.5 & 29.4 & 21.3 \\
\hline MS 2053 & $0.583^{\mathrm{c}}$ & $20: 56: 21.0$ & $-04: 37: 49$ & 18.7 & 18.0 & 5.1 \\
\hline MACS J0025.4 & $0.584^{\mathrm{a}}$ & $00: 25: 29.9$ & $-12: 22: 45$ & 14.3 & 19.7 & 12.3 \\
\hline MACS J2129.4 & $0.589^{\mathrm{a}}$ & $21: 29: 25.7$ & $-07: 41: 31$ & 13.2 & 21.3 & 15.2 \\
\hline MACS J0647.7 & $0.591^{\mathrm{a}}$ & $06: 47: 49.7$ & $+70: 14: 56$ & 11.7 & 22.0 & 14.4 \\
\hline MACS J0744.8 & $0.698^{\mathrm{a}}$ & $07: 44: 52.3$ & $+39: 27: 27$ & 16.3 & 20.6 & 13.3 \\
\hline MS 1054 & $0.831^{\mathrm{g}}$ & $10: 56: 58.5$ & $-03: 37: 34$ & 18.3 & 13.9 & 17.4 \\
\hline CL J0152.7 & $0.833^{\mathrm{a}}$ & $01: 52: 41.1$ & $-13: 58: 07$ & 9.3 & 23.4 & 10.2 \\
\hline CL J1226.9 & $0.888^{\mathrm{a}}$ & $12: 26: 57.9$ & $+33: 32: 49$ & 11.8 & 22.9 & 13.0 \\
\hline
\end{tabular}

Notes. The Bolocam X-ray/SZ (BOXSZ) cluster sample of 45 objects. The columns give the name, redshift, X-ray centroid coordinates (J2000), total Bolocam integration time, rms noise level of the SZ images, and peak S/N in the optimally filtered images (see Sayers et al. 2012a for details of how the peak $\mathrm{S} / \mathrm{N}$ and optimal filter are determined). The superscripts denote the reference for the redshifts, with ${ }^{\mathrm{a}}$ Mantz et al. (2010), ${ }^{\mathrm{b}}$ Allen et al. (2008), ${ }^{\mathrm{c}}$ Maughan et al. (2012), ${ }^{\mathrm{d}}$ Richard et al. (2010), ${ }^{\mathrm{e}}$ Jee et al. (2007), ${ }^{\mathrm{f}}$ Christensen et al. (2012), and ${ }^{\mathrm{g}}$ Tran et al. (2007).

by an above average amount of data from disturbed systems at larger radii. At these large radii, data and simulations indicate that there is little or no difference in the pressure profiles based on morphological classification, so we expect that any resulting bias in our results will be minor (Borgani et al. 2004; Nagai et al. 2007; Piffaretti \& Valdarnini 2008; A10). We address this issue in more detail in Section 5.1, where we examine the average pressure profiles of our cool-core and disturbed subsamples separately and find that they are consistent outside of $\simeq 0.15 R_{500}$ given our measurement uncertainties.

\section{DATA REDUCTION}

\subsection{Bolocam}

Bolocam is a 144-element bolometric imaging photometer, and from 2003-2012 it served as the long-wavelength facility camera for the Caltech Submillimeter Observatory (CSO). Bolocam covers an 8 arcmin diameter circular field of view (FOV) and has PSFs with 58 arcsec FWHMs (Glenn et al. 1998; Haig et al. 2004). The SZ-emission-weighted band center of our data is $140 \mathrm{GHz}$. All of the cluster images were obtained by 
Table 2

Details of the Chandra ACIS Observations Used in This Work

\begin{tabular}{|c|c|c|c|c|c|c|}
\hline \multirow{2}{*}{$\begin{array}{l}\text { Cluster } \\
\text { A383 }\end{array}$} & \multicolumn{3}{|c|}{ Date } & \multirow{2}{*}{$\begin{array}{r}\text { ObsID } \\
524\end{array}$} & \multirow{2}{*}{$\begin{array}{c}\text { Mode } \\
\text { VFAINT }\end{array}$} & \multirow{2}{*}{$\frac{\text { Exp. (ks) }}{8.2}$} \\
\hline & 2000 & Sep & 8 & & & \\
\hline & 2000 & Nov & 16 & 2320 & VFAINT & 16.8 \\
\hline & 2000 & Nov & 16 & 2321 & FAINT & 17.2 \\
\hline A611 & 2001 & Nov & 3 & 3194 & VFAINT & 33.8 \\
\hline AS1063 & 2004 & May & 17 & 4966 & VFAINT & 23.8 \\
\hline \multirow[t]{2}{*}{ A 370} & 1999 & Oct & 22 & 515 & FAINT & 67.8 \\
\hline & 2006 & Nov & 26 & 7715 & VFAINT & 6.6 \\
\hline \multirow[t]{2}{*}{ ZWCL 0024} & 2000 & Sep & 6 & 929 & FAINT & 33.8 \\
\hline & 2006 & Nov & 28 & 7717 & VFAINT & 7.0 \\
\hline MACS J0416.1 & 2009 & Jun & 7 & 10446 & VFAINT & 14.3 \\
\hline MACS J0451.9 & 2005 & Jan & 8 & 5815 & VFAINT & 9.6 \\
\hline \multirow[t]{4}{*}{ MACS J0329.6 } & 2001 & Nov & 25 & 3257 & VFAINT & 8.1 \\
\hline & 2002 & Dec & 24 & 3582 & VFAINT & 19.8 \\
\hline & 2004 & Dec & 6 & 6108 & VFAINT & 35.3 \\
\hline & 2006 & Dec & 3 & 7719 & VFAINT & 7.1 \\
\hline \multirow[t]{4}{*}{ MACS J1311.0 } & 2002 & Dec & 15 & 3258 & VFAINT & 14.9 \\
\hline & 2005 & Apr & 20 & 6110 & VFAINT & 63.0 \\
\hline & 2007 & Mar & 3 & 7721 & VFAINT & 6.6 \\
\hline & 2007 & Dec & 9 & 9381 & VFAINT & 24.1 \\
\hline \multirow[t]{2}{*}{ MS 2053} & 2000 & May & 13 & 551 & FAINT & 37.7 \\
\hline & 2001 & Oct & 7 & 1667 & VFAINT & 36.0 \\
\hline MS 1054 & 2000 & Apr & 21 & 512 & FAINT & 75.8 \\
\hline CL J0152.7 & 2000 & Sep & 8 & 913 & FAINT & 29.2 \\
\hline \multirow[t]{3}{*}{ CL J1226.9 } & 2000 & Jul & 31 & 932 & VFAINT & 9.8 \\
\hline & 2003 & Jan & 27 & 3180 & VFAINT & 26.1 \\
\hline & 2004 & Aug & 7 & 5014 & VFAINT & 25.3 \\
\hline
\end{tabular}

Notes. Cluster name, Chandra observation date, observation ID number, observing mode used, and clean exposure time for clusters presented in this work that did not appear in Mantz et al. (2010). We refer the reader to that work for details of the other Chandra observations.

scanning the CSO in a Lissajous pattern (Kovacs et al. 2006), with an amplitude of 4 arcmin and an average scan speed of $\simeq 4 \operatorname{arcmin} \mathrm{s}^{-1}$. These scans result in images with tapered coverage extending to a radius of $\simeq 12$ arcmin, with the coverage dropping to half its peak value at a radius of $\simeq 5 \operatorname{arcmin}$. For ease of analysis, we have made $14 \times 14$ arcmin square maps for each cluster.

Our Bolocam data reduction largely followed the procedure described in detail in Sayers et al. (2011), and we therefore briefly summarize that procedure below. First, we use frequent observations of bright compact objects to obtain pointing corrections accurate to 5 arcsec. Additionally, we made nightly observations of Uranus, Neptune, and/or other secondary calibrators to obtain flux calibration accurate to $5 \%$ (Griffin \& Orton 1993; Sandell 1994; Sayers et al. 2012b). To remove noise from atmospheric fluctuations, we subtract the FOV-average signal at each time sample in the time-ordered data (TOD) and also highpass filter the TOD at a characteristic frequency of $250 \mathrm{mHz}$. This process also attenuates the astronomical signals in our data, and we characterize this filtering as follows. First, for each observation, we insert a model cluster profile in our TOD, process these model-plus-data TOD through our reduction pipeline, and create a map. We then subtract the data-only map to produce a noiseless image of the model after going through our data processing pipeline. The result is compared to the original input model to obtain a complex-valued two-dimensional map-space transfer function.

To characterize the non-astronomical noise in our images we form jackknife realizations of the data by multiplying a randomly selected subset of half of the data by -1 prior to

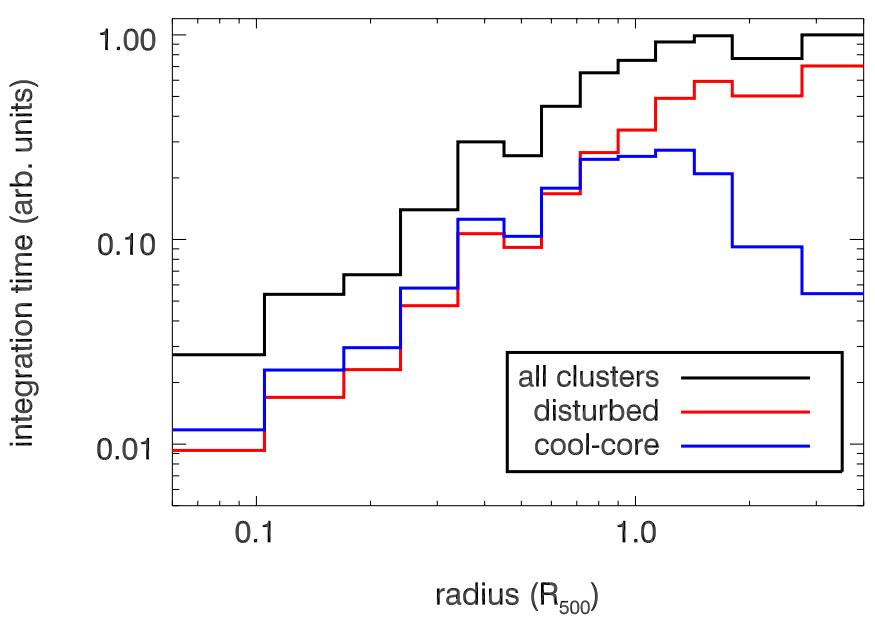

Figure 1. Total Bolocam integration time for BOXSZ clusters as a function of scaled radius for the same 13 radial bins given in the left column of Table 4 . The black line denotes the full BOXSZ sample, the red line denotes the disturbed subsample, and the blue line denotes the cool-core subsample.

(A color version of this figure is available in the online journal.)

binning the data into a map. For each cluster we formed 1000 such jackknife maps. To each of these maps we then added a Gaussian random realization of the $140 \mathrm{GHz}$ astronomical sky based on the power spectrum measurements made by the SPT, which cover all of the angular scales probed by our data (Keisler et al. 2011; Reichardt et al. 2012). Each of these 1000 astronomical signal realizations was processed through our data reduction pipeline so that it was filtered identically to our real data. We have verified that the above noise model is statistically equivalent to measurements of blank sky made with Bolocam (Sayers et al. 2011).

In addition, for 11 of our clusters we have subtracted individual bright point sources selected from the NVSS $1.4 \mathrm{GHz}$ catalog (Condon et al. 1998). We refer the reader to Sayers et al. (2013) for a full description of these sources, most of which are near the cluster centers. Since all of these sources are below our $140 \mathrm{GHz}$ detection limit, we have extrapolated spectral fits to 1.4 and $30 \mathrm{GHz}$ data. In almost all cases, the uncertainty on these extrapolated flux densities is $\simeq 30 \%$, limited by the intrinsic scatter in the extrapolation. We subtracted all of the sources with extrapolated flux densities $>0.5 \mathrm{mJy}$. This source brightness threshold was chosen to ensure that contamination of the cluster signal from point sources is $<1 \%$. To account for our uncertainty in the flux density of these subtracted point sources, we add a model of each point source, multiplied by a random value drawn from a Gaussian distribution described by our uncertainty on the extrapolated source flux density, to each of the 1000 noise realizations for a given cluster observation.

Furthermore, we detect a total of six bright point sources in our $140 \mathrm{GHz}$ data, all of which were subtracted for this analysis (Sayers et al. 2013). For these sources, we refit the point source model to each of our 1000 jackknife realizations and added a point source template to each of our 1000 noise realizations based on the dispersion of these fits.

\subsection{X-Ray Data}

X-ray luminosities, temperatures, and masses for the BOXSZ clusters appearing in Mantz et al. (2010, hereafter M10) are taken from that work. For the other clusters, these quantities were derived from archival Chandra data following the same 
Table 3

X-Ray Properties of the BOXSZ Sample

\begin{tabular}{|c|c|c|c|c|c|c|c|c|c|}
\hline Cluster & $\begin{array}{l}R_{500} \\
(\mathrm{Mpc})\end{array}$ & $\begin{array}{c}L_{500} \\
\left(10^{44} \mathrm{erg} \mathrm{s}^{-1}\right)\end{array}$ & $\begin{array}{c}M_{500} \\
\left(10^{14} M_{\odot}\right)\end{array}$ & $\begin{array}{c}k T \\
(\mathrm{keV})\end{array}$ & $\begin{array}{c}P_{500} \\
\left(10^{-3} \mathrm{keV} \mathrm{cm}^{-3}\right)\end{array}$ & $L_{\text {rat }}$ & $\begin{array}{c}w_{500} \\
\left(10^{-2}\right)\end{array}$ & Cool Core & Disturbed \\
\hline A2204 & $1.46 \pm 0.07$ & $17.9 \pm 1.6$ & $10.3 \pm 1.5$ & $8.6 \pm 0.6$ & $4.56 \pm 1.26$ & $0.35 \pm 0.07$ & $0.13 \pm 0.04$ & $\checkmark$ & \\
\hline A383 & $1.11 \pm 0.06$ & $6.0 \pm 0.2$ & $4.7 \pm 0.8$ & $5.4 \pm 0.2$ & $2.85 \pm 0.87$ & $0.28 \pm 0.03$ & $0.19 \pm 0.03$ & $\checkmark$ & \\
\hline A209 & $1.53 \pm 0.08$ & $8.6 \pm 0.3$ & $12.6 \pm 1.9$ & $8.2 \pm 0.7$ & $5.64 \pm 1.60$ & $0.07 \pm 0.02$ & $0.50 \pm 0.17$ & & \\
\hline A963 & $1.25 \pm 0.06$ & $6.5 \pm 0.2$ & $6.8 \pm 1.0$ & $6.1 \pm 0.3$ & $3.74 \pm 1.04$ & $0.15 \pm 0.02$ & $0.22 \pm 0.11$ & & \\
\hline A1423 & $1.35 \pm 0.10$ & $6.2 \pm 0.4$ & $8.7 \pm 2.0$ & $5.8 \pm 0.6$ & $4.45 \pm 1.67$ & $0.13 \pm 0.03$ & $0.76 \pm 0.19$ & & \\
\hline A2261 & $1.59 \pm 0.09$ & $12.0 \pm 0.4$ & $14.4 \pm 2.6$ & $6.1 \pm 0.3$ & $6.32 \pm 2.02$ & $0.20 \pm 0.02$ & $0.85 \pm 0.08$ & $\checkmark$ & \\
\hline A2219 & $1.74 \pm 0.08$ & $15.5 \pm 0.8$ & $18.9 \pm 2.5$ & $10.9 \pm 0.5$ & $7.62 \pm 1.98$ & $0.07 \pm 0.02$ & $0.18 \pm 0.13$ & & \\
\hline A267 & $1.22 \pm 0.07$ & $5.8 \pm 0.2$ & $6.6 \pm 1.1$ & $7.1 \pm 0.7$ & $3.79 \pm 1.15$ & $0.08 \pm 0.02$ & $2.68 \pm 1.26$ & & $\checkmark$ \\
\hline RX J2129.6 & $1.28 \pm 0.07$ & $9.9 \pm 0.5$ & $7.7 \pm 1.2$ & $6.3 \pm 0.6$ & $4.23 \pm 1.23$ & $0.25 \pm 0.03$ & $0.52 \pm 0.14$ & $\checkmark$ & \\
\hline A1835 & $1.49 \pm 0.06$ & $21.1 \pm 0.6$ & $12.3 \pm 1.4$ & $9.0 \pm 0.2$ & $5.94 \pm 1.39$ & $0.36 \pm 0.02$ & $0.23 \pm 0.02$ & $\checkmark$ & \\
\hline A697 & $1.65 \pm 0.09$ & $14.4 \pm 0.8$ & $17.1 \pm 2.9$ & $10.9 \pm 1.1$ & $7.72 \pm 2.36$ & $0.08 \pm 0.02$ & $0.60 \pm 0.45$ & & \\
\hline A611 & $1.24 \pm 0.06$ & $7.5 \pm 0.4$ & $7.4 \pm 1.1$ & $6.8 \pm 0.3$ & $4.45 \pm 1.25$ & $0.16 \pm 0.03$ & $0.56 \pm 0.10$ & & \\
\hline MS 2137 & $1.06 \pm 0.04$ & $11.1 \pm 0.4$ & $4.7 \pm 0.6$ & $4.7 \pm 0.4$ & $3.42 \pm 0.87$ & $0.40 \pm 0.03$ & $0.39 \pm 0.05$ & $\checkmark$ & \\
\hline AS1063 & $1.76 \pm 0.09$ & $30.8 \pm 1.6$ & $22.2 \pm 3.4$ & $10.9 \pm 0.5$ & $10.14 \pm 2.90$ & $0.16 \pm 0.04$ & $0.75 \pm 0.15$ & & \\
\hline MACS J1931.8 & $1.34 \pm 0.07$ & $19.7 \pm 1.0$ & $9.9 \pm 1.6$ & $7.5 \pm 1.4$ & $5.95 \pm 1.77$ & $0.40 \pm 0.04$ & $0.35 \pm 0.09$ & $\checkmark$ & \\
\hline MACS J1115.8 & $1.28 \pm 0.06$ & $14.5 \pm 0.5$ & $8.6 \pm 1.2$ & $9.2 \pm 1.0$ & $5.45 \pm 1.47$ & $0.28 \pm 0.02$ & $0.27 \pm 0.05$ & $\checkmark$ & \\
\hline MACS J1532.9 & $1.31 \pm 0.08$ & $19.8 \pm 0.7$ & $9.5 \pm 1.7$ & $6.8 \pm 1.0$ & $5.89 \pm 1.87$ & $0.38 \pm 0.03$ & $0.28 \pm 0.15$ & $\checkmark$ & \\
\hline A 370 & $1.40 \pm 0.08$ & $8.6 \pm 0.4$ & $11.7 \pm 2.1$ & $7.3 \pm 0.5$ & $6.89 \pm 2.19$ & $0.04 \pm 0.01$ & $4.90 \pm 2.00$ & & $\checkmark$ \\
\hline MACS J1720.3 & $1.14 \pm 0.07$ & $10.2 \pm 0.4$ & $6.3 \pm 1.1$ & $7.9 \pm 0.7$ & $4.65 \pm 1.45$ & $0.26 \pm 0.02$ & $0.24 \pm 0.06$ & $\checkmark$ & \\
\hline ZWCL 0024 & $1.00 \pm 0.11$ & $2.3 \pm 0.1$ & $4.4 \pm 1.6$ & $5.9 \pm 0.9$ & $3.70 \pm 1.89$ & $0.10 \pm 0.03$ & $2.53 \pm 0.41$ & & $\checkmark$ \\
\hline MACS J2211.7 & $1.61 \pm 0.07$ & $24.0 \pm 1.2$ & $18.1 \pm 2.5$ & $14.0 \pm 2.7$ & $9.52 \pm 2.55$ & $0.19 \pm 0.03$ & $0.88 \pm 0.13$ & $\checkmark$ & \\
\hline MACS J0429.6 & $1.10 \pm 0.05$ & $10.9 \pm 0.6$ & $5.8 \pm 0.8$ & $8.3 \pm 1.6$ & $4.48 \pm 1.20$ & $0.33 \pm 0.04$ & $0.39 \pm 0.07$ & $\checkmark$ & \\
\hline MACS J0416.1 & $1.27 \pm 0.15$ & $8.1 \pm 0.5$ & $9.1 \pm 2.0$ & $8.2 \pm 1.0$ & $6.25 \pm 2.28$ & $0.04 \pm 0.02$ & $2.02 \pm 1.06$ & & $\checkmark$ \\
\hline MACS J0451.9 & $1.12 \pm 0.06$ & $6.7 \pm 0.5$ & $6.3 \pm 1.1$ & $6.7 \pm 1.0$ & $4.97 \pm 1.55$ & $0.08 \pm 0.03$ & $1.93 \pm 0.80$ & & $\checkmark$ \\
\hline MACS J1206.2 & $1.61 \pm 0.08$ & $21.1 \pm 1.1$ & $19.2 \pm 3.0$ & $10.7 \pm 1.3$ & $10.59 \pm 3.07$ & $0.15 \pm 0.03$ & $0.72 \pm 0.11$ & & \\
\hline MACS J0417.5 & $1.69 \pm 0.07$ & $29.1 \pm 1.5$ & $22.1 \pm 2.7$ & $9.5 \pm 1.1$ & $11.70 \pm 2.88$ & $0.19 \pm 0.03$ & $3.01 \pm 0.07$ & $\checkmark$ & $\checkmark$ \\
\hline MACS J0329.6 & $1.19 \pm 0.06$ & $13.4 \pm 0.4$ & $7.9 \pm 1.3$ & $6.3 \pm 0.3$ & $5.96 \pm 1.79$ & $0.33 \pm 0.02$ & $1.40 \pm 0.26$ & $\checkmark$ & $\checkmark$ \\
\hline MACS J1347.5 & $1.67 \pm 0.08$ & $42.2 \pm 1.1$ & $21.7 \pm 3.0$ & $10.8 \pm 0.8$ & $11.71 \pm 3.13$ & $0.39 \pm 0.02$ & $0.59 \pm 0.04$ & $\checkmark$ & \\
\hline MACS J1311.0 & $0.93 \pm 0.04$ & $7.5 \pm 0.2$ & $3.9 \pm 0.5$ & $6.0 \pm 0.3$ & $3.99 \pm 1.01$ & $0.19 \pm 0.01$ & $0.22 \pm 0.08$ & $\checkmark$ & \\
\hline MACS J2214.9 & $1.39 \pm 0.08$ & $13.9 \pm 0.6$ & $13.2 \pm 2.3$ & $9.6 \pm 0.8$ & $9.12 \pm 2.85$ & $0.10 \pm 0.02$ & $1.30 \pm 0.29$ & & $\checkmark$ \\
\hline MACS J0257.1 & $1.20 \pm 0.06$ & $12.1 \pm 0.5$ & $8.5 \pm 1.3$ & $9.9 \pm 0.9$ & $6.82 \pm 1.95$ & $0.12 \pm 0.02$ & $0.46 \pm 0.13$ & & \\
\hline MACS J0911.2 & $1.22 \pm 0.06$ & $7.5 \pm 0.3$ & $9.0 \pm 1.2$ & $6.6 \pm 0.6$ & $7.09 \pm 1.85$ & $0.05 \pm 0.01$ & $0.89 \pm 0.64$ & & \\
\hline MACS J0454.1 & $1.31 \pm 0.06$ & $15.7 \pm 0.6$ & $11.5 \pm 1.5$ & $9.1 \pm 0.5$ & $8.79 \pm 2.26$ & $0.07 \pm 0.01$ & $2.27 \pm 1.50$ & & $\checkmark$ \\
\hline MACS J1423.8 & $1.09 \pm 0.05$ & $14.0 \pm 0.5$ & $6.6 \pm 0.9$ & $6.9 \pm 0.3$ & $6.12 \pm 1.62$ & $0.37 \pm 0.03$ & $0.31 \pm 0.15$ & $\checkmark$ & \\
\hline MACS J1149.5 & $1.53 \pm 0.08$ & $17.2 \pm 0.7$ & $18.7 \pm 3.0$ & $8.5 \pm 0.6$ & $12.28 \pm 3.62$ & $0.05 \pm 0.01$ & $1.64 \pm 1.23$ & & $\checkmark$ \\
\hline MACS J0018.5 & $1.47 \pm 0.08$ & $18.0 \pm 0.9$ & $16.5 \pm 2.5$ & $9.1 \pm 0.4$ & $11.33 \pm 3.22$ & $0.06 \pm 0.02$ & $0.67 \pm 0.14$ & & \\
\hline MACS J0717.5 & $1.69 \pm 0.06$ & $25.0 \pm 0.9$ & $24.9 \pm 2.7$ & $11.8 \pm 0.5$ & $14.90 \pm 3.39$ & $0.05 \pm 0.01$ & $2.55 \pm 1.26$ & & $\checkmark$ \\
\hline MS 2053 & $0.82 \pm 0.06$ & $2.8 \pm 0.1$ & $3.0 \pm 0.5$ & $4.4 \pm 0.6$ & $3.86 \pm 1.17$ & $0.07 \pm 0.02$ & $1.02 \pm 0.31$ & & $\checkmark$ \\
\hline MACS J0025.4 & $1.12 \pm 0.04$ & $9.1 \pm 0.4$ & $7.6 \pm 0.9$ & $6.5 \pm 0.5$ & $7.18 \pm 1.73$ & $0.03 \pm 0.01$ & $0.65 \pm 0.50$ & & \\
\hline MACS J2129.4 & $1.25 \pm 0.06$ & $13.7 \pm 0.6$ & $10.6 \pm 1.4$ & $8.6 \pm 0.7$ & $9.03 \pm 2.34$ & $0.08 \pm 0.02$ & $1.51 \pm 0.69$ & & $\checkmark$ \\
\hline MACS J0647.7 & $1.26 \pm 0.06$ & $14.1 \pm 0.6$ & $10.9 \pm 1.6$ & $11.5 \pm 1.1$ & $9.23 \pm 2.57$ & $0.10 \pm 0.02$ & $0.62 \pm 0.29$ & & \\
\hline MACS J0744.8 & $1.26 \pm 0.06$ & $18.9 \pm 0.6$ & $12.5 \pm 1.6$ & $8.1 \pm 0.4$ & $11.99 \pm 3.05$ & $0.16 \pm 0.02$ & $1.60 \pm 0.11$ & & $\checkmark$ \\
\hline MS 1054 & $1.07 \pm 0.07$ & $12.4 \pm 0.7$ & $9.0 \pm 1.3$ & $12.0 \pm 1.4$ & $11.90 \pm 3.28$ & $0.02 \pm 0.01$ & $6.62 \pm 2.47$ & & $\checkmark$ \\
\hline CL J0152.7 & $0.97 \pm 0.26$ & $7.3 \pm 0.6$ & $7.8 \pm 3.0$ & $6.5 \pm 0.9$ & $10.86 \pm 5.74$ & $0.01 \pm 0.01$ & $8.22 \pm 1.02$ & & $\checkmark$ \\
\hline CL J1226.9 & $1.00 \pm 0.05$ & $14.0 \pm 0.5$ & $7.8 \pm 1.1$ & $12.0 \pm 1.3$ & $11.84 \pm 3.21$ & $0.10 \pm 0.02$ & $0.95 \pm 0.31$ & & \\
\hline
\end{tabular}

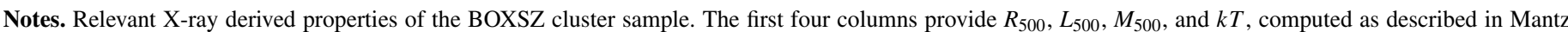

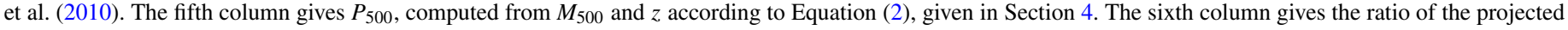

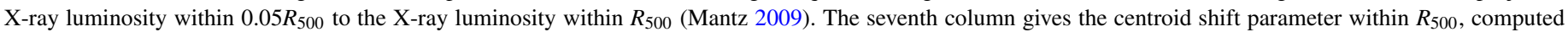

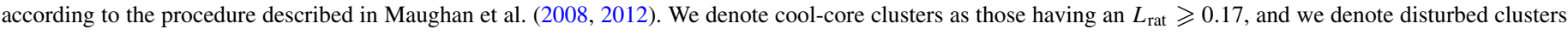
as those having $w_{500} \geqslant 0.01$.

procedure as was used in M10, and we refer the reader there for full details. Briefly, the archival data were reprocessed using ciao $^{10}$ (version 4.1.1; CALDB 4.1.2), including removal of bad pixels, corrections for cosmic ray afterglows and charge transfer inefficiency, and application of standard grade and status filters, using appropriate time-dependent gain and calibration products. Soft-band surface brightness profiles were extracted and were scaled by a global factor to agree with the final ROSAT

\footnotetext{
10 http://cxc.harvard.edu/ciao/
}

flux calibration. ${ }^{11}$ These profiles were then used to derive cluster luminosity, projected luminosity ratio (Equation (1)), and gas mass. Values of total mass and $R_{500}$ were derived for a reference $h=0.7, \Omega_{m}=0.3$, flat $\Lambda \mathrm{CDM}$ cosmology using the derived gas mass profiles and the universal gas mass fraction measured by Allen et al. (2008). Spectra were extracted from an annulus

\footnotetext{
11 This scaling of the Chandra data is described in detail in Section 2.2.4 of M10 and was motivated by the primary goal of that analysis, which was to relate Chandra-derived masses to ROSAT survey fluxes. For consistency with the results of M10, we have retained this scaling in our present analysis.
} 
covering radii between $0.15 R_{500}$ and $R_{500}$ and fit in xspec ${ }^{12}$ to provide a global temperature measurement. For the lowest redshift cluster (A2204), the luminosity and gas mass analyses used ROSAT Position Sensitive Proportional Counter data, since the Chandra field of view does not comfortably encompass $R_{500}$, and the average temperature measured from $A S C A$ data by Horner et al. (1999) was adopted.

In addition, we used the Chandra data to constrain the centroid shift parameter $w_{500}$. This analysis was described in Maughan et al. (2012), and we refer the reader to that manuscript for additional details. Centroid shifts measure the standard deviation of the projected separation between the X-ray peak and the centroid as a function of projected radius $R_{p}<R_{500}$. Following the same approach as Poole et al. (2006), $w_{500}$ was computed from a series of circular apertures with initial and final radii $R_{p}=R_{500}$ and $R_{p}=0.05 R_{500}$ respectively, decreasing in size by $0.05 R_{500}$ in each iteration. To this end, we used background-subtracted images, appropriately divided by the exposure map to eliminate instrumental artifacts such as chip gaps and vignetting. All point sources were excluded from the analysis; however, extended sources were left untouched as these may be associated with some of the cluster substructure.

\section{PRESSURE DEPROJECTIONS}

In order to determine physical pressures from our SZ data (which are measured in units of a cosmic microwave background (CMB) fluctuation temperature), we use the equations described by Sunyaev \& Zel'dovich (1972) with the relativistic corrections given by Itoh et al. (1998). For the relativistic corrections, we have assumed that the ICM of each cluster is isothermal, with a temperature equal to the spectroscopic X-ray temperature given in Table 3. Although the true temperature profiles are expected to vary by factors of $\simeq 2$ over the radii probed by our data, we note that the relativistic corrections are $\lesssim 10 \%$ for these clusters in our observing band. Therefore, even if the ICM temperature varies by a factor of two over the radial range probed by our data, then our isothermal approximation will produce less than a $5 \%$ bias on the pressures that we derive.

Due to the high-pass filtering applied to the Bolocam data to remove atmospheric noise, it is not possible to directly deproject pressure profiles from the standard Bolocam images. We have demonstrated the ability to deconvolve the effects of this high pass filter (Sayers et al. 2011), and we are able to produce unbiased images that could in principal be deprojected. However, to prevent unphysical numerical artifacts from appearing in the deconvolved images, we would need to reduce the size of our images from 14 to $10 \mathrm{arcmin}$. Since we are interested in information about the pressure profiles at large radii, we do not want to accept this loss of information.

Consequently, we compute our deprojected pressure profiles as follows. First, we select a set of discrete radial points (referred to as radial bins) at which we would like to compute the spherically symmetric deprojected pressure. Next, we create a smooth and continuous pressure profile by connecting these radial bins with a power-law interpolation. This smooth pressure profile is then projected into a two dimensional SZ model. For the projection, we assume that the profile is equal to zero outside of $5 R_{500}$, and we use a power-law extrapolation to estimate the pressure profile beyond our largest radial bin and inside $5 R_{500}$. We then filter this SZ model with both the signal transfer

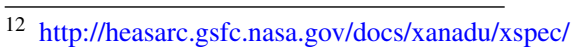

function of our data and the Bolocam PSF, and then compare the filtered SZ model to our data map.

We determine the best-fit pressure deprojections using the MPFITFUN generalized least squares (GLS) fitting algorithm (Markwardt 2009) under the simplifying assumption that the noise covariance matrix of our SZ maps is diagonal. As described in Sayers et al. (2011), a diagonal noise covariance matrix is a very good, but not perfect, description of our SZ map data. Consequently, we compute all of our uncertainties using the 1000 different noise realizations described in Section 3.1. First, we add a cluster model, equal to the best-fit deprojection of our data, separately to each of these 1000 noise realizations. We then fit a pressure deprojection to each of these 1000 model-plusnoise realizations, and quantify all of our uncertainties based on the spread of these 1000 fits. By design, this procedure fully accounts for all of the characteristics of our noise to quantify our uncertainties for a deprojection of a cluster with a pressure profile described by our best-fit deprojection. One possible bias in this approach is that it only quantifies the uncertainties for a particular cluster shape, and the true cluster profile might differ from our best-fit profile. Consequently, we tested this effect by computing uncertainties for our deprojection fits using both the best-fit profile to our cluster sample (Section 5.1) and the bestfit profile found by A10 as the input shapes added to our 1000 noise realizations. We find that the uncertainties recovered using an A10 profile differ from the recovered uncertainties using our best-fit profile by an $\mathrm{rms}$ of $7.7 \%$. If the uncertainties were identical, then we would expect an rms difference of $4.5 \%$ due to our finite number of noise realizations. This indicates that our uncertainties on the recovered deprojection depend slightly on the exact shape of the cluster pressure profile. However, since the true cluster pressure profile is likely to be similar to our bestfit profile, and since the variation in recovered uncertainties is similar to our precision in estimating them due to our finite number of noise realizations, we do not attempt to account for the cluster-shape dependence of our recovered uncertainties in our analysis. Finally, we have verified that our GLS algorithm adequately samples the parameter space by showing that it is robust to our choice of initial conditions, and that we are able to recover an input cluster candidate with minimal bias (see Figure 2).

Throughout this work we compare and average the pressure profiles from multiple clusters. Therefore, prior to any deprojection, we have scaled the radial coordinate of each cluster by $R_{500}$ and the pressure amplitude by

$$
P_{500}=\left(3.68 \times 10^{-3} \frac{\mathrm{keV}}{\mathrm{cm}^{3}}\right)\left(\frac{M_{500}}{10^{15} M_{\odot}}\right)^{\alpha_{P}} E(z)^{8 / 3},
$$

where $E^{2}(z)=\Omega_{M}(1+z)^{3}+\Omega_{\Lambda}$ and $\alpha_{P}=2 / 3$ is the nominal scaling predicted by self-similar hydrostatic equilibrium models (Nagai et al. 2007; A10). ${ }^{13}$ We note that our definition of $P_{500}$ is valid for the electron pressure of the ICM and not the total pressure.

We have determined the average pressure profile of the full BOXSZ sample (along with the disturbed and cool-core subsamples) via a simultaneous joint deprojection of multiple clusters. In this approach, a single deprojected profile in units

\footnotetext{
13 Unlike, e.g., A10 or P12, our joint deprojections do not include any corrections to the value of $\alpha_{P}$, mainly because such corrections have negligible effects on our results. For example, correcting the value of $\alpha_{P}$ from $2 / 3$ to our best-fit $\alpha_{P}=0.49$ found in Section 5.2 causes the pressure values in the individual joint deprojection bins for the full sample to change by $\lesssim 2 \%$.
} 


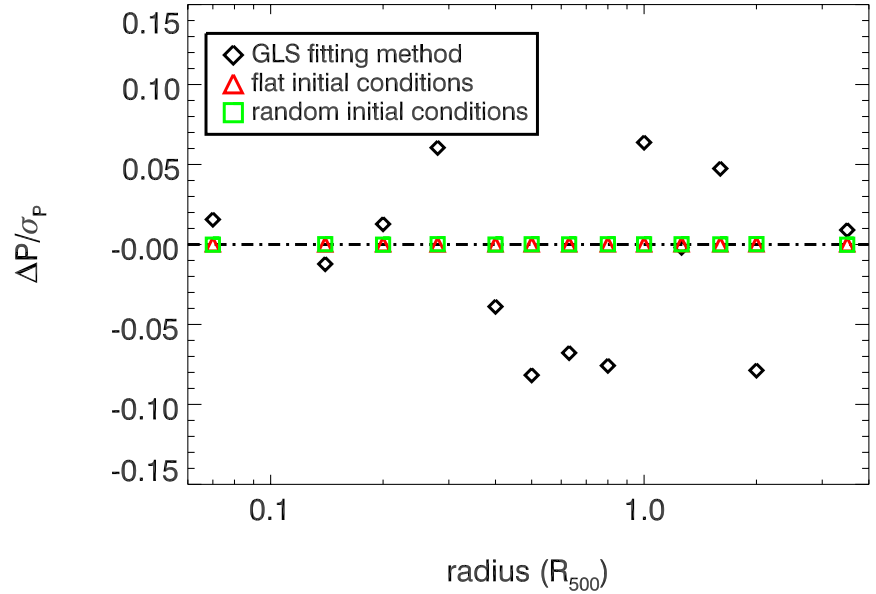

Figure 2. GLS fitting bias divided by the uncertainty for each radial bin in the joint pressure deprojection of the full BOXSZ sample. The black diamonds show the difference between the input pressure profile and the average recovered pressure profile using our GLS algorithm. The red triangles and green squares show the additional bias associated with choosing different starting values for the pressure bins relative to our default starting values equal to the best-fit model from A10 (for the red triangles all of the starting values were set equal to $P_{500}$, and for the green squares all of the starting values were set equal to a randomly drawn value between 0 and $\left.10 P_{500}\right)$. In all cases, the bias is quasi-negligible when added in quadrature with the uncertainty (note that the typical $\mathrm{S} / \mathrm{N}$ per bin is $\simeq 5$, so the absolute bias is $\simeq 2 \%$ ). This result shows that our fitting method is approximately unbiased and that it is robust to our choice of initial conditions (i.e., it adequately explores the parameter space).

(A color version of this figure is available in the online journal.)

of $P_{500}$ and $R_{500}$ is constrained by the data from an arbitrary number of clusters using the GLS algorithm described above. Pressure deprojections for the full BOXSZ sample, the coolcore subsample, and the disturbed subsample are shown in Figure 3 and numerical values are given in Table 4. For the deprojection of the full BOXSZ sample, we have used 13 approximately logarithmically spaced bins between $0.07 R_{500}$ and $3.5 R_{500}$. These deprojections extend beyond the cluster virial radius (typically near $2 R_{500}$; Umetsu et al. 2011), although only in two deprojection bins, each with a signal-to-noise ratio $(\mathrm{S} / \mathrm{N}) \simeq 1.5$. Due to the smaller number of clusters in the two subsamples, we have used seven bins spanning the same radial range in each case. We note that, for many of the clusters in our sample, particularly the preferentially higher redshift disturbed clusters, the innermost radial bin(s) is(are) inside of Bolocam's PSF half-maximum radius of 29 arcsec (in the most extreme case $0.07 R_{500}$ is $\simeq 8$ arcsec). However, we have shown that even for the disturbed subsample we are able to obtain unbiased fits to the innermost bins for deprojections of model input clusters, indicating that our joint deprojections are sensitive to the shape of the pressure profile at these sub-PSF-sized scales.

We determine the covariance matrix of our deprojected pressure profiles directly from the set of 1000 deprojections of model-plus-noise data. The off-diagonal elements of the covariance matrix are in general non-zero and positive at large radii, indicating significant positive correlations between most of those deprojection bins (see Figure 4). This can be understood as a consequence of the high-pass filtering applied to our data, which results in SZ images that are sensitive to the large-scale shape of the pressure profile (i.e., $d P / d R$ ) but not its absolute normalization. In contrast, the high-pass filtering does not have a significant effect on the deprojection bins at small radii, and adjacent bins at small radii tend to have strong negative correlations. These large correlations, which

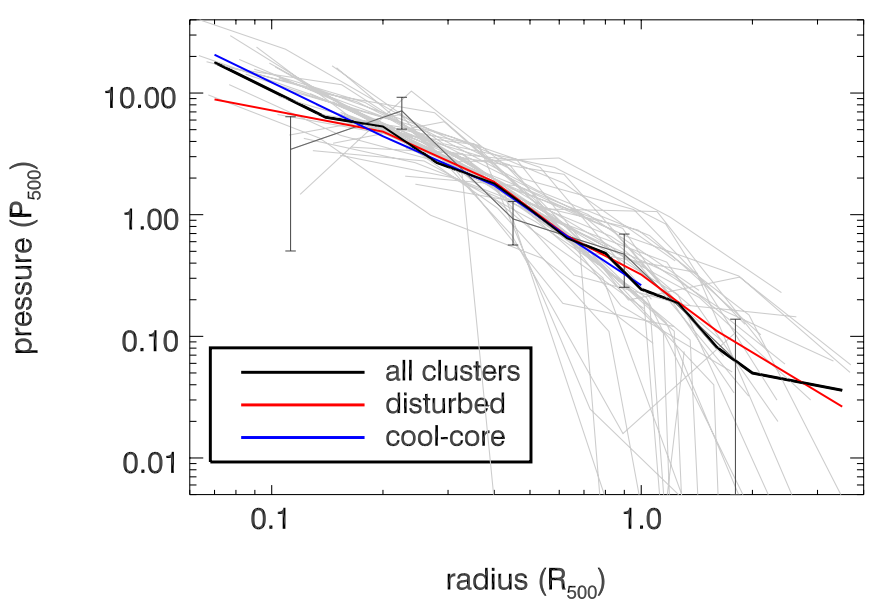

Figure 3. Pressure deprojection for the full BOXSZ sample (thick black line), the disturbed subsample (thick red line), and the cool-core subsample (thick blue line). Plotted as thin gray lines are the pressure deprojections for each of the 45 clusters in the BOXSZ sample. Each individual cluster was deprojected into five radial bins located at $0.5,1.0,2.0,4.0$, and 8.0 arcmin in order to fully sample the spatial dynamic range of our images. One of the clusters is shown as a darker gray line with error bars at each deprojection bin to indicate the typical uncertainty on each of the individual cluster deprojections. A significant amount of the variation in the individual cluster profiles is due to measurement uncertainty, but we do find an additional cluster-to-cluster dispersion that is described in Section 5.2. Note that, in all cases, the correlations between bins are large and varied and must be accounted for in any fit or interpretation of the data.

(A color version of this figure is available in the online journal.)

Table 4

Pressure Deprojections

\begin{tabular}{lccc}
\hline \hline $\begin{array}{l}\text { Radius } \\
\left(R_{500}\right)\end{array}$ & $\begin{array}{c}\text { All Clusters } \\
\left(P_{500}\right)\end{array}$ & $\begin{array}{c}\text { Disturbed } \\
\left(P_{500}\right)\end{array}$ & $\begin{array}{c}\text { Cool Core } \\
\left(P_{500}\right)\end{array}$ \\
\hline 0.07 & $(1.79 \pm 0.30) \times 10^{+1}$ & $(0.87 \pm 0.16) \times 10^{+1}$ & $(2.07 \pm 0.14) \times 10^{+1}$ \\
0.14 & $(6.32 \pm 1.48) \times 10^{0}$ & $\ldots$ & $\ldots$ \\
0.20 & $(5.31 \pm 1.11) \times 10^{0}$ & $(4.81 \pm 0.43) \times 10^{0}$ & $(4.41 \pm 0.29) \times 10^{0}$ \\
0.28 & $(2.68 \pm 0.38) \times 10^{0}$ & $\ldots$ & $\ldots$ \\
0.40 & $(1.81 \pm 0.23) \times 10^{0}$ & $(1.86 \pm 0.15) \times 10^{0}$ & $(1.74 \pm 0.15) \times 10^{0}$ \\
0.50 & $(1.11 \pm 0.13) \times 10^{0}$ & $\ldots$ & $\ldots$ \\
0.63 & $(6.41 \pm 1.01) \times 10^{-1}$ & $(6.69 \pm 0.94) \times 10^{-1}$ & $(6.64 \pm 0.93) \times 10^{-1}$ \\
0.80 & $(4.82 \pm 0.69) \times 10^{-1}$ & $\ldots$ & $\ldots$ \\
1.00 & $(2.44 \pm 0.61) \times 10^{-1}$ & $(3.22 \pm 0.62) \times 10^{-1}$ & $(2.63 \pm 0.56) \times 10^{-1}$ \\
1.26 & $(1.87 \pm 0.45) \times 10^{-1}$ & $\ldots$ & $\ldots$ \\
1.60 & $(0.81 \pm 0.39) \times 10^{-1}$ & $(1.11 \pm 0.40) \times 10^{-1}$ & $(\leqslant 0.62) \times 10^{-1}$ \\
2.00 & $(4.99 \pm 3.72) \times 10^{-2}$ & $\ldots$ & $\ldots$ \\
3.50 & $(3.60 \pm 2.63) \times 10^{-2}$ & $(2.64 \pm 2.12) \times 10^{-2}$ & $(\leqslant 8.13) \times 10^{-2}$ \\
\hline
\end{tabular}

Notes. Deprojected pressure profiles for the full BOXSZ sample, the disturbed subsample, and the cool-core subsample. The error bars give the square root of the diagonal elements of the covariance matrix. The best-fit pressure in the two outermost bins of the cool-core deprojection is consistent with zero, and so we instead list $68 \%$ confidence level upper limits on those values.

exist for both the jointly and independently deprojected profiles, must be accounted for in any interpretation of our results. As we noted above, our estimation of the correlation matrix assumes a particular cluster pressure profile. We again tested this dependence by computing a correlation matrix using both our best-fit profile and the best-fit profile of A10 as inputs, and we find that the rms difference between the recovered elements of the covariance matrices for the two input profiles is $4.3 \%$. This value matches the expected variation due to our finite number of noise realizations, and therefore indicates that our 


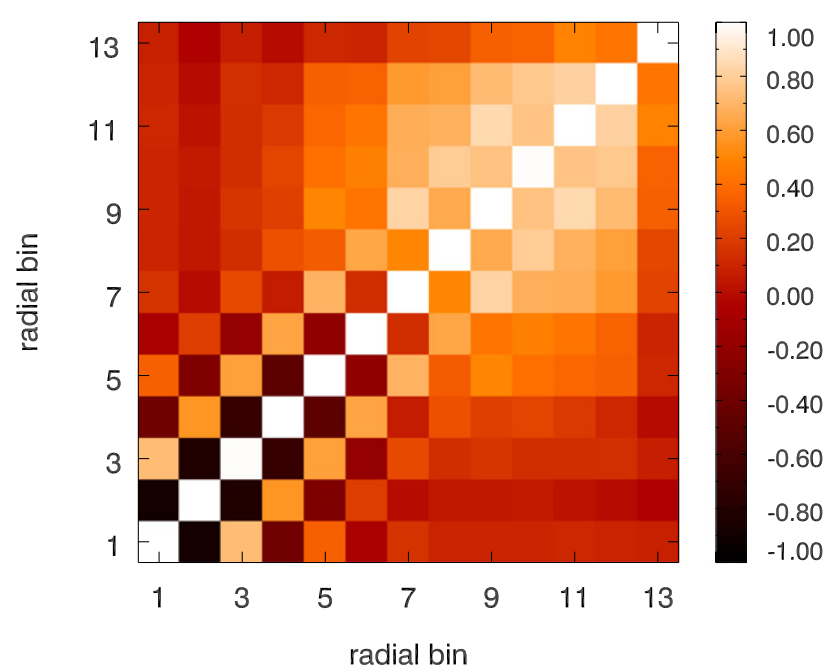

Figure 4. Correlation matrix for the pressure deprojection of the full data set into 13 radial bins from $0.07 R_{500}$ to $3.5 R_{500}$ (see Table 4 for the exact radius of each bin). At large radii the adjacent bins have significant positive correlations due to the high pass filtering applied to the data, while at small radii the highpass filtering has little effect on the data, and consequently there are significant anti-correlations between adjacent bins at those radii.

(A color version of this figure is available in the online journal.)

method for estimating the correlation matrix is independent of our underlying cluster pressure profile.

In addition to the joint deprojections described above, we also deprojected the pressure profiles of each cluster individually using an identical technique. However, due to the significantly varied range of scaled radii probed for each cluster, deprojecting all of the clusters at the same set of scaled radii is not ideal. Consequently, we have deprojected the individual clusters at fixed angular radii to ensure that they adequately constrain the profile over the scales probed by our data $(0.5,1.0,2.0,4.0$, and 8.0 arcmin). These individual deprojections are shown in Figure 3.

\section{AVERAGE PRESSURE PROFILES}

In this section we use the deprojections from Section 4 to constrain the average properties of cluster pressure profiles using two different techniques, and we briefly describe and compare those techniques here. In Section 5.1 we constrain parameterized pressure profiles to the joint deprojected profiles. Such fits have been performed in a number of previous analyses (e.g., A10 and P12), and these fits therefore enable us to directly compare our results to the results of those works. Since these fits ask a relatively simple question of our data (i.e., what is the measurement-noise-weighted average pressure profile), they also allow us to constrain the average profile of our sample with optimal signal to noise over a broad radial range, even for physically interesting subsets of the BOXSZ. In Section 5.2, we introduce a new technique for probing the ensemble behavior of cluster pressure profiles using a Gaussian process formalism. For this analysis we use the individual cluster pressure deprojections to simultaneously constrain an ensemble mean pressure profile, a covariance matrix describing the intrinsic scatter about this mean profile, and the mass scaling of the pressure profiles. This novel technique asks a more demanding set of questions of our data compared with a parametric fit of the average profile, and consequently limits us to constraints over a smaller radial range.

\subsection{Parameterized Pressure Profiles}

One method for characterizing the gross behavior of pressure profiles in our sample (or a subsample) is to fit a parameterized function to the data. For this analysis, we fit parametric models to our deprojected data, rather than directly to the map data, because fitting directly to the map data takes an inconveniently large amount of computing time, several days. As we describe in detail below, we found that parametric fits to our deprojected profiles, which require only a few minutes, were indistinguishable from parametric fits directly to the map data. We emphasize that there is no fundamental reason why the fits cannot be performed directly to the map data, and our choice to fit the deprojected profiles was motivated entirely by computational expediency.

To perform the parametric fits to our deprojected data, we make the simplifying assumption that the noise is Gaussian and therefore fully described by its covariance matrix. We applied Mardia's test of multivariate normality to confirm this assumption is correct (Mardia 1970). Mardia's skewness statistic has a limiting distribution equal to the $\chi^{2}$ distribution, and we find a $\chi^{2}$ per degree of freedom (DOF) equal to $\chi_{\text {red }}^{2}=$ $465 / 455$ (corresponding to a probability to exceed (PTE) of 0.36). Mardia's kurtosis statistic has a limiting distribution equal to the unit normal distribution, and we find a value of -0.96 (corresponding to a two-sided PTE of 0.34). Therefore, the noise properties of our deprojected profiles are consistent with Gaussian distributions. In addition, we directly fit a single generalized Navarro, Frenk, and White (NFW; Navarro et al. 1996) model to our map data, allowing all five parameters to vary. This model is described by

$$
\tilde{P}(X)=\frac{P_{0}}{\left(C_{500} X\right)^{\gamma}\left[1+\left(C_{500} X\right)^{\alpha}\right]^{(\beta-\gamma) / \alpha}},
$$

where $\tilde{P}(X)$ is the scaled pressure profile (in units of $P_{500}$ ), $X=R / R_{500}, P_{0}$ is the normalization, $C_{500}$ sets the radial scale, and $\alpha, \beta$, and $\gamma$ describe the power-law slope at moderate, large, and small radii (Nagai et al. 2007). Similarly to our deprojection procedure, we then determined our parameter uncertainties using fits to 1000 separate noise realizations. For comparison, we then performed a Markov chain Monte Carlo (MCMC) fit of the same model to our deprojected profile assuming a Gaussian covariance matrix. We find that the profiles recovered from fitting the map data directly and from fitting the deprojected data agree within $\lesssim 1 \%$ for the range of our deprojected data $\left(0.07 R_{500} \leqslant R \leqslant 3.5 R_{500}\right)$, indicating that the two methods recover consistent results. Finally, we have verified that there are no biases in our parametric model fits to either the map data or the deprojected data by performing both types of fits to a simulated cluster with a known profile. Specifically, we added a cluster with the best-fit A10 pressure profile to each of the 1000 noise realizations for each cluster, and repeated our analysis on each of these simulated data sets. We find that the average bestfit gNFW profiles to these data, both from direct fits to the map data and from fits to the joint deprojections, agree with the input A10 profile within $\lesssim 1 \%$ between $0.07 R_{500}$ and $3.5 R_{500}$. Since we find no significant difference between gNFW fits directly to the map data and gNFW fits obtained from the deprojected data, we obtained all of the results described below from fits to the deprojected data.

We find that our data do not constrain all five of the gNFW fit parameters within the physically allowed region. Specifically, our best-fit outer slope of $\beta=2.03$ implies an infinite total 


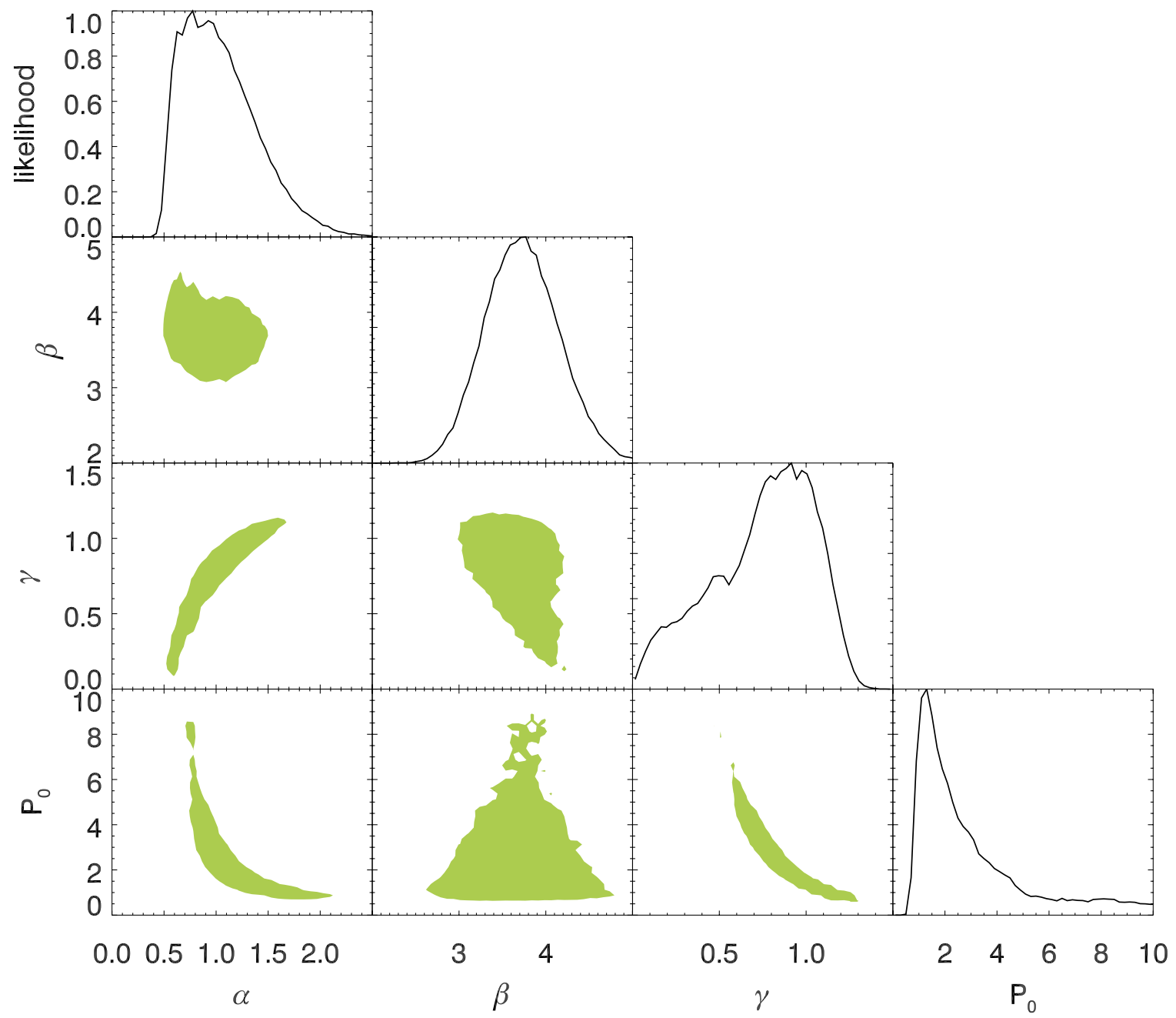

Figure 5. Two-parameter confidence regions (68\%) and one-parameter likelihoods for a gNFW fit to our joint deprojected profile of the full dataset. From left to right and top to bottom the plots show $\alpha, \beta, \gamma$, and $P_{0}$ with fixed $C_{500}=1.18$. The large degeneracies between the fit parameters are clearly seen, along with the corresponding lack of constraining power on any individual parameter. However, as shown in Figure 6, the overall pressure profile is tightly constrained.

(A color version of this figure is available in the online journal.)

pressure. ${ }^{14}$ As a result, we refit the data fixing the concentration parameter to $C_{500}=1.18$ (the best-fit value found by A10), obtaining a physically allowed outer slope of $\beta=3.67$. The quality of this four-parameter fit is $\operatorname{good}\left(\chi_{\text {red }}^{2}=1.0\right.$ for nine DOF), indicating that this four-parameter gNFW fit describes our data with a sufficient goodness of fit. We note that the actual pressure profile over the radial range constrained by our data $\left(0.07 R_{500} \lesssim R \lesssim 3.5 R_{500}\right)$ for this four-parameter $\mathrm{gNFW}$ fit is only slightly different from the five-parameter fit, indicating that the unphysical outer slope in the five-parameter gNFW fit is likely due to the finite radial extent of our data. Therefore, the best-fit gNFW model of the BOXSZ sample is given by

$$
\left[C_{500}, \alpha, \beta, \gamma, P_{0}\right]=[1.18,0.86,3.67,0.67,4.29],
$$

where the value of $C_{500}$ is in italics to emphasize that it was held fixed at 1.18 in our fits. Our choice to fix the value of $C_{500}$, rather than one of the other parameters, was motivated by the fact that all of the possible four-parameter gNFW fits have similar fit qualities, but any combination that varies

\footnotetext{
14 Here, and throughout this manuscript, we present best-fit parameter values without error estimates due to the large degeneracies between parameters, as illustrated in Figure 5.
}

both $C_{500}$ and $\beta$ results in an unphysical outer slope. The set of two-dimensional confidence regions for the four-parameter gNFW fit is illustrated in Figure 5, and the strong parameter degeneracies mentioned above are evident in these plots. To test for possible biases associated with our choice to fix $C_{500}$ to the best-fit value determined by A10, we also computed gNFW fits with the value of $C_{500}$ set to the best-fit value of P12, $C_{500}=1.81$, and to the best-fit value of Nagai et al. (2007), $C_{500}=1.30,{ }^{15}$ with the results shown in Table 5. Compared to our choice of $C_{500}=1.18$, we find that these values of $C_{500}$ result in similar fit qualities and profiles that differ by an rms of $4 \%(\mathrm{P} 12)$ and $1 \%$ (Nagai et al. 2007) over the radial range $0.07 R_{500} \leqslant R \leqslant 3.5 R_{500}$. Therefore, we conclude that our choice to fix $C_{500}$ to the best-fit value determined by A10 has little impact on our results.

We then fit the same four-parameter gNFW model to both the disturbed and cool-core subsamples of the BOXSZ cluster sample (see Table 5). In good agreement with previous results from A10 and P12, we find consistent average pressure profiles, given our measurement uncertainties, between these subsamples (and the full sample) at all intermediate and large radii

\footnotetext{
15 In their unpublished erratum, Nagai et al. find $C_{500}=1.3$ rather than the published value of $C_{500}=1.8$ (Nagai et al. 2007).
} 
Table 5

gNFW Fits to the Bolocam Cluster Sample

\begin{tabular}{|c|c|c|c|c|c|c|c|c|}
\hline$C_{500}$ & $\alpha$ & $\beta$ & $\gamma$ & $P_{0}\left(P_{500}\right)$ & $\chi_{\text {red }}^{2}$ & DOF & PTE & Notes \\
\hline \multicolumn{9}{|c|}{ All clusters } \\
\hline 1.81 & 1.33 & 4.13 & 0.31 & $6.54^{\star}$ & 4.7 & 12 & 0.00 & Best-fit shape from P12 \\
\hline 1.18 & 1.05 & 5.49 & 0.31 & $7.82^{\star}$ & 2.7 & 12 & 0.00 & Best-fit shape from A10 \\
\hline 1.18 & $0.86^{\star}$ & $3.67^{\star}$ & $0.67^{\star}$ & $4.29^{\star}$ & 1.0 & 9 & 0.44 & Best-fit from this work (using A10 value of $C_{500}$ ) \\
\hline 1.81 & $1.32^{\star}$ & $2.91^{\star}$ & $0.92^{\star}$ & $2.60^{\star}$ & 1.0 & 9 & 0.48 & Four-parameter fit using P12 value of $C_{500}$ \\
\hline 1.30 & $0.91^{\star}$ & $3.51^{\star}$ & $0.71^{\star}$ & $3.94^{\star}$ & 1.0 & 9 & 0.45 & Four-parameter fit using Nagai et al. (2007) value of $C_{500}$ \\
\hline $3.19^{\star}$ & $3.28^{\star}$ & $2.03^{\star}$ & $1.10^{\star}$ & $3.07^{\star}$ & 0.9 & 8 & 0.52 & Five-parameter fit, yields an unphysical outer slope $\beta$ \\
\hline \multicolumn{9}{|c|}{ Disturbed clusters } \\
\hline 1.18 & $0.90^{\star}$ & $5.22^{\star}$ & $0.02^{\star}$ & $17.28^{\star}$ & 3.1 & 4 & 0.02 & Best-fit from this work \\
\hline \multicolumn{9}{|c|}{ Cool-core clusters } \\
\hline 1.18 & $2.79^{\star}$ & $3.51^{\star}$ & $1.37^{\star}$ & $0.65^{\star}$ & 1.7 & 4 & 0.15 & Best-fit from this work \\
\hline
\end{tabular}

Notes. gNFW fits to the deprojected profiles computed for the BOXSZ sample. From left to right the columns give the concentration parameter relative to $R_{500}\left(C_{500}\right)$, the power-law slopes at intermediate, large, and small radii $(\alpha, \beta, \gamma)$, the normalization $P_{0}$ relative to $P_{500}$, the reduced $\chi^{2}$ of the fit, the degrees of freedom in the fit, the associated PTE, and any notes regarding the particular fit. The upper rows show fits to the deprojection of the full sample, and show parameter values and fit qualities when different numbers of parameters are varied. The lower two rows show the best-fit four-parameter models for the disturbed and cool-core subsamples. In all cases, stars denotes parameters that were varied in the fit. In the first two rows, we fit the models of P12 and A10, varying only the normalization. In all subsequent rows, the fixed parameters were set to the values found in A10. We find that varying four parameters provides a sufficient goodness of fit to the data, which is only marginally improved when all five parameters are varied. However, varying all five parameters results in a profile with an unphysically small outer slope $(\beta=2.03)$, and we therefore take the four-parameter fit as the best description of our data.

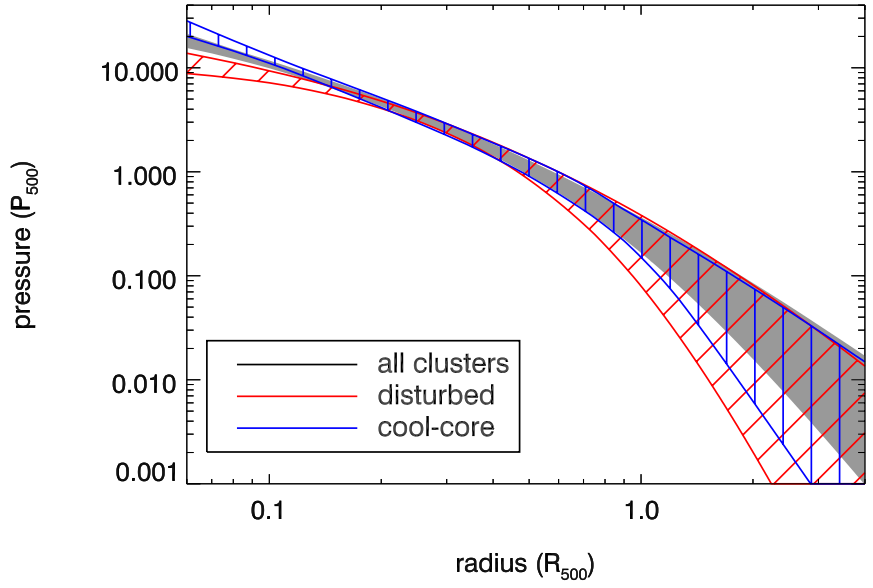

Figure 6. gNFW parameterized fits to the Bolocam data, varying four parameters of the gNFW model ( $C_{500}$ was fixed to 1.18$)$. The bands indicate the maximum and minimum pressure values as a function of radius, bounding $68 \%$ of the MCMC fits. The plot shows fits to the full BOXSZ, the disturbed subsample, and the cool-core subsample. The pressure profile appears to be independent of cluster morphology at $R \gtrsim 0.15 R_{500}$, but the cool-core clusters have higher pressures than the disturbed clusters at smaller radii.

(A color version of this figure is available in the online journal.)

$\left(R \gtrsim 0.15 R_{500}\right)$. At smaller radii, the profiles clearly diverge, and at those radii the cool-core clusters have a higher pressure than the disturbed clusters (see Figure 6). ${ }^{16}$ Although the overall pressure profiles for the full BOXSZ sample, the cool-core subsample, and the disturbed subsample are quite similar at most radii, we note that the best-fit gNFW parameters are quite different, further emphasizing the large degeneracies between these parameters. Furthermore, the fit quality of the gNFW model is slightly worse for both of the two subsamples compared with the full sample, with PTEs of 0.02 and 0.15 for the disturbed

\footnotetext{
${ }^{16}$ As we described in detail in Section 2, there is an asymmetry in the redshift distributions of the cool-core and disturbed subsamples. Consequently, we cannot definitively rule out redshift evolution as the cause of the discrepancy in the pressure profiles at small radii. However, given that A10 observed a similar difference in the inner pressure profiles of disturbed and cool-core systems for a sample of low-redshift clusters, the differences we observe are likely due to morphology rather than redshift evolution.
}

and cool-core fits, respectively, compared with a PTE of 0.44 for the full-sample fit.

Due to the large degeneracies between the parameters in the gNFW model, it is difficult to quantify the differences between our best-fit gNFW model and those found in previous analyses via a direct comparison of the fit parameters (Nagai et al. 2007; A10; Plagge et al. 2010; P12). Consequently, we have compared the profiles resulting from our $\mathrm{gNFW}$ fits to the $\mathrm{gNFW}$ profiles found by $\mathrm{A} 10$ and $\mathrm{P} 12$ over the approximate radial range constrained by all three datasets $\left(0.05 R_{500} \lesssim R \lesssim 4 R_{500}\right.$, see Figures 7 and 8). We, in general, find excellent agreement between our pressure profiles and those found in these previous analyses, regardless of morphological classification ${ }^{17}$ (e.g., the cool-core profile from our analysis is in good agreement with the cool-core profile of A10). We do note that our disturbed and cool-core systems indicate slight differences within $0.3 R_{500}$ compared with the corresponding results of A10, and our overall average profile indicates slightly higher pressures at $R \lesssim 0.1 R_{500}$ and at $R \gtrsim 1.0 R_{500}$ compared with the results of A10. Our overall average profile also shows higher pressure at small radii compared with the results of P12. Our results are therefore more similar to simulation-derived results at small radii, which also show higher pressures than found by A10 and P12 (A10; Borgani et al. 2004; Nagai et al. 2007; Piffaretti \& Valdarnini 2008; P12).

The overall good agreement between our best-fit gNFW profiles and the results derived in previous analyses provides further evidence that the average cluster pressure profile is approximately universal (at least within our measurement uncertainties on the average profile, which are $\simeq 10-20 \%$ inside $R_{500}$ ). This is especially true given the large differences in the median redshifts $(\langle z\rangle=0.12,0.15$, and 0.42$)$, median masses $\left(\left\langle M_{500}\right\rangle=3\right.$, 6 , and $\left.9 \times 10^{14} M_{\odot}\right)$ and data types (X-ray/simulation, X-ray/ SZ, SZ-only) for the A10, P12, BOXSZ samples. As another consistency check between our data and the results of A10 and P12, we fit each of their best-fit gNFW models to our data,

\footnotetext{
17 P12 only present results for cool-core and non-cool-core subsamples, and we therefore take their non-cool-core results to be representative of disturbed systems.
} 


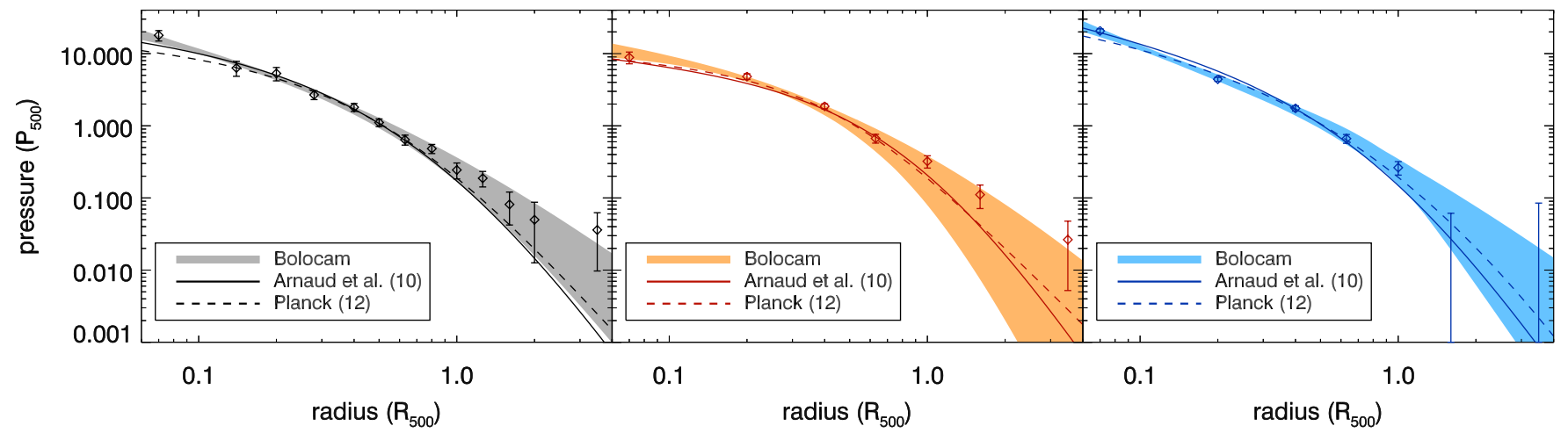

Figure 7. gNFW parameterized fits to the BOXSZ sample, varying four parameters of the gNFW model. From left to right the three plots show the Bolocam fit to the full sample, disturbed subsample, and cool-core subsample as points with error bars and with shaded regions representing the $68.3 \%$ confidence region for the gNFW fits (we note that gNFW fits with five free parameters are slightly more consistent with the measured data at large radius, but, as described in the text, these fits were discarded because they produce unphysical outer slope). The best-fit parameterizations given in A10 and P12 are overlaid as thin and dashed lines (P12 did not fit a disturbed subsample, so we overlay their non-cool-core fit in the center plot). The A10 fits relied on the REXCESS sample of 33 low- $z$ clusters $(z<0.2)$ observed with $X M M$-Newton within $R_{500}$ and results from simulations outside $R_{500}$. The P12 fits relied on a sample of 62 Planck-selected clusters at $\langle z\rangle \simeq 0.15$, and used XMM-Newton data to constrain the inner portion of the profile and Planck data to constrain the outer portion of the profile. Our fits use Bolocam SZ data for a sample of 45 higher redshift clusters $(0.15 \leqslant z \leqslant 0.89)$.

(A color version of this figure is available in the online journal.)
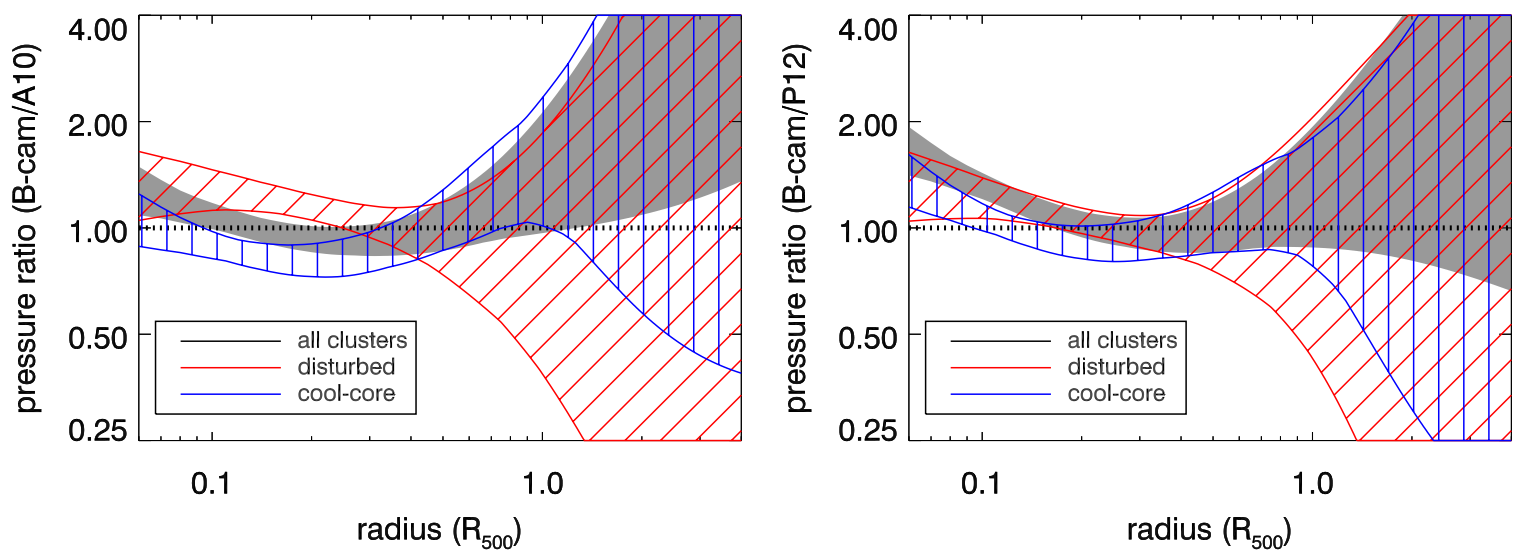

Figure 8. Confidences regions (68.3\%) for the ratio of our best-fit four-parameter gNFW fits to the best-fit four-parameter gNFW fits from A10 (left) and P12 (right). In both cases the agreement is generally good in the regions that are well constrained by all three datasets $\left(0.1 R_{500} \lesssim R \lesssim 1.0 R_{500}\right)$. However, the fit to the full BOXSZ sample shows hints of higher pressure than the A10 fit at both large and small radii, and hints of higher pressure than the P12 fit at small radius.

(A color version of this figure is available in the online journal.)

allowing only the normalization to be a free parameter. Although the fit quality is poor in both cases, we find normalizations consistent with both results (7.82 compared to 8.40 for the A10 fit and 6.54 compared to 6.41 for the P12 fit). ${ }^{18}$ This implies that the average total pressure of our sample is consistent with the average total pressure found in those analyses, further showing the approximate universality of cluster pressure profiles and the good agreement between SZ and X-ray measurements of those profiles. In addition, we note that two other analyses show good agreement with the results of A10 (and consequently our results as well). Sun et al. (2011) analyzed Chandra data for a set of 43 low redshift groups and found an average pressure profile that is within $1 \sigma$ of the A10 profile over nearly all of its range $\left(0.01 R_{500} \lesssim R \lesssim R_{500}\right)$. Plagge et al. (2010) used SPT SZ data for a set of 15 moderate redshift clusters to constrain a gNFW

\footnotetext{
18 Although these single-parameter gNFW fits do not suffer from the same degeneracies seen in the multi-parameter fits, we forgo error estimates because the poor fit quality calls into question the accuracy of such estimates. However, the fits indicate that our uncertainty on the normalization is likely to be dominated by our $5 \%$ flux calibration uncertainty. Since our values differ from the A10 and P12 values by $7 \%$ and $2 \%$, we can conclude that the normalizations found by all three datasets are consistent.
}

profile, finding shape parameters that are statistically consistent with the A10 values (3/4 parameters agree within $1 \sigma$, and the fourth agrees within $2 \sigma$ ).

In addition to fitting gNFW models to our data, we also fit a $\beta$-model of the form

$$
\tilde{P}(X)=\frac{P_{0}}{\left(1+\left(X / R_{c}\right)^{2}\right)^{3 \beta / 2}},
$$

where $P_{0}$ is the pressure normalization, $R_{c}$ is the core radius, and $\beta$ is the power-law slope (Cavaliere \& Fusco-Femiano 1976, 1978). Fitting the full BOXSZ sample, we find best-fit parameters of $R_{c}=0.11$ (relative to $R_{500}$ ), $\beta=0.61$ and $P_{0}=18.9$. We find $\chi_{\text {red }}^{2}=1.5$ for $10 \mathrm{DOF}$, which gives a PTE of 0.13 and indicates a somewhat worse fit compared with the gNFW model. The fit appears to be largely driven by the higher $\mathrm{S} / \mathrm{N}$ data at small radii, which explains why the bestfit value of $\beta$ is more similar to $\mathrm{X}$-ray derived results from fits to the inner regions of clusters $(\beta \simeq 2 / 3$; e.g., Jones \& Forman 1984; Arnaud 2009) than those derived from X-ray surface brightness profiles at large radii (e.g., Vikhlinin et al. 1999; Maughan et al. 2008) or from SZ data extending to large radii ( $\beta \simeq 0.85-1.05$; e.g., Hallman et al. 2007; Plagge et al. 
2010). Given the superior fit quality of the gNFW model, along with the known shortcomings of the $\beta$-model (e.g., Mohr et al. 1999; Hallman et al. 2007), we do not explore the $\beta$-model in any additional detail.

\subsection{Gaussian Process Description of the Ensemble Properties of the Pressure Profiles}

Jointly fitting distinct subsets of clusters provides some information on the differences among cluster pressure profiles as a function of radius, but does not directly probe the intrinsic scatter among these profiles. Investigating the scatter instead requires a model for the ensemble of profiles to be fit to the data from individual clusters. Here we adopt arguably the simplest such model, describing scaled cluster pressure profiles as a Gaussian process (for an introduction to Gaussian processes; see, e.g., Rasmussen \& Williams 2006).

In this approach, the ensemble of profiles is modeled by (1) a mean scaled pressure profile as a function of scaled radius, $\bar{P}(x),{ }^{19}$ and (2) a covariance function, $\Sigma(x, y)$, encoding the intrinsic scatter about $\bar{P}(x)$ as a function of radius. Mathematically, the likelihood for a single realization of $P(x)$ to have a set of scaled pressures $\left\{P_{i}\right\}$ at scaled radii $\left\{X_{i}\right\}$ is proportional to

$$
\frac{\exp \left(-\frac{1}{2} z^{\mathrm{T}} S^{-1} z\right)}{|S|^{1 / 2}}
$$

where $z_{i}=P_{i}-\bar{P}\left(X_{i}\right)$ and $S_{i, j}=\Sigma\left(X_{i}, X_{j}\right)$, for any pair of radial values $X_{i}$ and $X_{j}$. Thus, the diagonal covariance terms, $\Sigma(x, x)$ dictate the marginal intrinsic scatter among profiles at a given radius; while off-diagonal terms, $\Sigma(x, y)$ with $x \neq y$, determine whether realizations of the profile tend to be shifted coherently with respect to $\bar{P}$ (positive values), or tend to cross $\bar{P}$ (negative values). In addition to the mean profile and scatter, we fit simultaneously for the mass dependence of the pressure normalization via the parameter $\alpha_{P}$ in Equation (2). ${ }^{20}$

The individually deprojected cluster profiles from Section 4 will be used to constrain this model. However, those profiles are each constrained at different scaled radii, presenting a significant complication to the analysis. To simplify the problem, we interpolate the individual profiles to a set of five common scaled radii, logarithmically spaced between $0.1 R_{500}$ and $2.0 R_{500}$. The radial range probed here is smaller than that covered by the sample as a whole, reflecting the fact that data from a sufficient number of clusters must exist at each radius in order to constrain the scatter. The interpolation was accomplished as follows. First, we generate a multivariate normal draw of the pressures at the deprojection radii using the measured mean values and measurement error covariance matrices of the individual cluster deprojections from Section 4. We then interpolate these pressures to the set of five common scaled radii using a power-law interpolation (recall that our deprojections assumed power-law behavior between the deprojection radii). This process is repeated many times in order to constrain the mean pressures and the measurement error covariance matrices at the common scaled radii. Since the individual profiles do

\footnotetext{
19 Note that this ensemble mean profile is conceptually different from the average parameterized profiles fit in Section 5.1. The former describes the average profile accounting for the presence of intrinsic scatter, i.e., the center of an ensemble of profiles at a given radius, while the latter assumes that all cluster profiles are described by a single function, with residuals between the model and data entirely due to known measurement uncertainties.

20 Including a free power of $E(z)$ has a negligible effect on our results in this section. Since the data constrain this evolution term very poorly, we did not investigate such an evolution term further.
}

not cover the entire range $0.1<X<2$, each cluster provides information at only a subset of the final radii. The result of this procedure is that the mean profile and covariance function can be compactly parameterized by five pressure values (for $\bar{P}$ ) and the independent elements of a $5 \times 5$ covariance matrix (for $\Sigma$ ), corresponding to the common scaled radii, where otherwise we would have been forced to assume a particular functional form for $\Sigma$. is

Thus, the complete log-likelihood used to constrain the model

$$
\ln \mathcal{L}=\sum_{j}-\frac{1}{2}\left[z_{j}^{\mathrm{T}}\left(S+U_{j}\right)^{-1} z_{j}+\ln \left|S+U_{j}\right|\right],
$$

where the sum is over clusters, and $U_{j}$ is the measurement error covariance matrix for the interpolated, scaled pressure profile of the $j$ th cluster. The parameter space for this model was explored using MCMC, adopting flat priors on all 21 free parameters. Maximum-likelihood confidence intervals for each parameter are displayed in Table 6, and Figure 9 shows the recovered pressure profile and fractional scatter as a function of radius. The reduced $\chi^{2}$ of our data with respect to the best-fitting model is 1.02 for $137 \mathrm{DOF}$, indicating that the Gaussian process description of the ensemble of profiles provides a sufficient goodness of fit. In particular, while the difference in gNFW profile fits of cool-core and disturbed clusters is evident at $0.1 R_{500}$ in Figure 6 , its modest statistical significance at that radius is reflected in this analysis, where a simple Gaussian scatter is seen to be a satisfactory model for our data. ${ }^{21}$

We constrain the mass scaling from Equation (2) to be $\alpha_{P}=0.49_{-0.08}^{+0.10}$, which is shallower than the value of $2 / 3$ predicted by self-similar hydrostatic equilibrium scalings (Kaiser 1986; A10). Under the assumption that the pressure profile shape is independent of mass, the integrated SZ signal $Y$, or its X-ray analog $Y_{X}$, scale with mass according to a powerlaw slope $\alpha_{Y}=\alpha_{P}+1$. Our value of $\alpha_{P}$ therefore implies a $Y-M$ scaling with a power-law slope of 1.49 , in good agreement with the $Y_{X}-M$ power-law slope of $1.48 \pm 0.04$ found by M10 using an identical method for constraining cluster masses from Chandra X-ray data. ${ }^{22}$ These results are inconsistent with those of A10, who measured a $Y_{X}-M$ scaling with $\alpha_{Y}=1.78 \pm 0.06$, implying $\alpha_{P}=0.78$. We speculate that this discrepancy in values of $\alpha_{P}$ is due to differences in mass estimation between A10 and our work (which uses masses determined according to M10). Indeed, Rozo et al. (2012a, 2012b) have noted that the difference in $Y_{X^{-}} M$ slopes between A10 and M10 is consistent with being due to a systematic disagreement in mass estimates, after accounting for instrumental calibration. Comparable disagreements in X-ray temperature-mass relation slopes, loosely correlated with hydrostatic mass calibration techniques, were also pointed out by Mantz \& Allen (2011). In addition, as described in Section 4, we emphasize that our results for the shape of the average pressure profile are approximately independent of the exact mass scaling, and therefore should not be affected by any of these discrepancies in mass determinations.

\footnotetext{
21 We also attempted to constrain the intrinsic scatter within the disturbed and cool-core subsamples. Unfortunately, neither subsample provides enough data at these small radii for us to constrain ensemble models for the individual subsamples.

22 A separate analysis of the BOXSZ sample, which accounts for selection effects and uses directly integrated cylindrical $Y$ values within $R_{2500}$, also finds a similar slope for the scaling of $Y$ versus $M$ (N. G. Czakon et al. 2013, in preparation).
} 

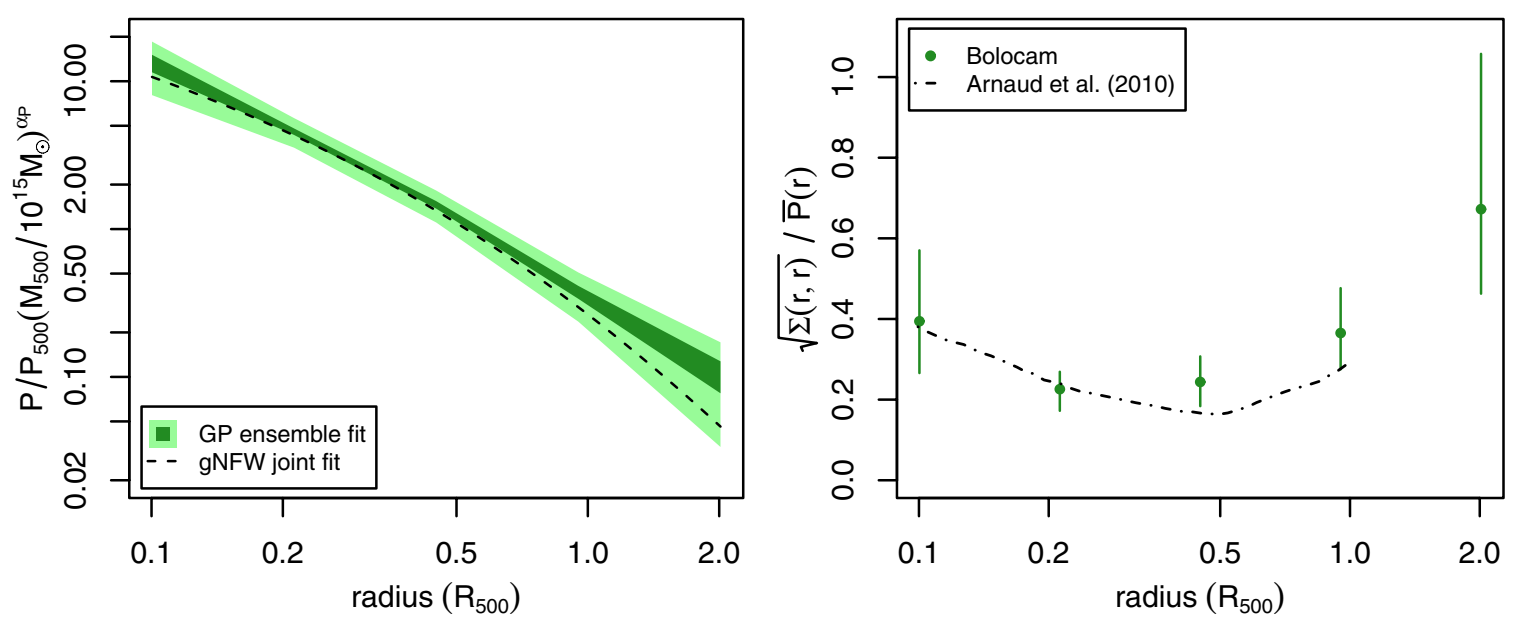

Figure 9. Left: the dark, inner shaded region shows the $68.3 \%$ confidence posterior for the mean pressure profile determined from our Gaussian process analysis, while the light, outer region indicates the best-fit marginal intrinsic scatter at each radius (the square root of the diagonal elements of the covariance matrix). At all radii, the uncertainty on the mean function is smaller than the corresponding nominal intrinsic scatter. The dashed line shows the best gNFW joint fit, which assumes that a single profile describes all clusters, and differs slightly from the Gaussian process fit, which includes intrinsic scatter. Right: the fractional intrinsic scatter (diagonal covariances scaled by the best-fitting mean profile) as a function of radius. At all radii, zero intrinsic scatter is excluded at $>95.4 \%$ confidence. The intrinsic scatter estimated by A10 from X-ray data is shown as the dot-dashed line, and is in good agreement with our results at $R<R_{500}$.

(A color version of this figure is available in the online journal.)

Table 6

Gaussian Process Fit Parameters

\begin{tabular}{|c|c|c|c|c|c|}
\hline radius $\left(R_{500}\right)$ & 0.100 & 0.212 & 0.449 & 0.951 & 2.013 \\
\hline \multicolumn{6}{|c|}{ Mean scaled pressure profile, $\bar{P}(X)$} \\
\hline & $13.3_{-1.8}^{+1.8}$ & $4.59_{-0.26}^{+0.20}$ & $1.47_{-0.09}^{+0.08}$ & $0.37_{-0.04}^{+0.04}$ & $0.102_{-0.025}^{+0.025}$ \\
\hline \multicolumn{6}{|c|}{ Intrinsic scatter covariance matrix, $\Sigma\left(X_{i}, X_{j}\right)$} \\
\hline 0.100 & $27.5_{-15.0}^{+30.0}$ & $2.75_{-2.00}^{+2.50}$ & $0.85_{-0.80}^{+0.90}$ & $0.22_{-0.35}^{+0.45}$ & $0.07_{-0.24}^{+0.26}$ \\
\hline 0.212 & & $1.08_{-0.45}^{+0.45}$ & $0.19_{-0.11}^{+0.17}$ & $0.04_{-0.04}^{+0.07}$ & $0.02_{-0.04}^{+0.04}$ \\
\hline 0.449 & & & $0.13_{-0.05}^{+0.08}$ & $0.037_{-0.018}^{+0.024}$ & $0.009_{-0.012}^{+0.018}$ \\
\hline 0.951 & & & & $0.019_{-0.008}^{+0.013}$ & $0.0038_{-0.0055}^{+0.0065}$ \\
\hline 2.013 & & & & & $0.0047_{-0.0025}^{+0.0070}$ \\
\hline
\end{tabular}

Notes. Gaussian process fit parameters describing the mean pressure profile of our sample and the intrinsic scatter about this profile. The fit also includes an overall mass scaling, constrained to $\alpha_{P}=0.49_{-0.08}^{+0.10}$; see the text in Section 5.2. All of the diagonal elements of the covariance matrix are different from zero at greater than $95.4 \%$ significance, indicating that we detect non-zero intrinsic scatter at all radii. Most of the off-diagonal elements are consistent with zero, indicating that our mass and redshift scaling largely accounts for any evolution in the normalization of the pressure profiles.

Our fit detects the presence of non-zero intrinsic scatter among profiles (diagonal covariance terms) at a significant level ( $>95.4 \%$ confidence) at all radii, with the fractional intrinsic scatter minimized at radii $\simeq 0.2 R_{500}-0.5 R_{500}$. The off-diagonal covariance terms become consistent with zero at large radial separations (i.e., large $\left.\left|X_{1}-X_{2}\right|\right)$, indicating that our pressure scaling has effectively removed the principal mass and redshift dependence of the profiles. The adequacy of our simple scaling over a wide redshift and mass range further confirms that cluster pressure profiles are approximately universal, with fractional intrinsic scatter away from the universal profile at the $\simeq 20 \%-40 \%$ level.

In addition, given our measurement uncertainties, the intrinsic scatter of our high mass and moderate redshift BOXSZ sample is consistent with that found using X-ray data within $R_{500}$ for the lower mass and lower redshift clusters in the REXCESS sample (A10; Figure 9) and for low redshift groups (Sun et al. 2011), supporting the proposition that the intrinsic scatter among pressure profiles is relatively independent of mass and redshift. Given the large redshift range spanned by the BOXSZ sample, this comparison to results at lower redshifts also indicates that the inexact redshift scalings of the Kaiser (1986) relations do not introduce a significant amount of intrinsic scatter among pressure profiles (Kravtsov \& Borgani 2012). Furthermore, similar to the findings of P12, our results imply that the intrinsic scatter continues to increase at radii larger than those probed previously by X-ray analyses $\left(\gtrsim R_{500}\right)$.

We note that the Gaussian process approach employed in this section provides a compact framework to obtain simultaneous constraints on the ensemble mean profile, its scaling relations, and its radius-dependent intrinsic scatter. In the present analysis, we have made some assumptions for computational expediency, namely that the measurement errors and intrinsic scatter are described well as Gaussian; however, neither of these assumptions is necessarily required. Looking forward, such probabilistic models in general are attractive because they allow straightforward and quantitative comparisons of clusters from different observed samples or from simulations. For example, 
given such a description of simulated cluster profiles, it would be straightforward to test whether observed profiles are consistent with the simulations using simple $\chi^{2}$ statistics.

\section{SUMMARY}

We have examined the pressure profiles determined from Bolocam SZ observations of the 45 clusters in the BOXSZ sample. This sample spans a large range in redshift $(0.15 \leqslant z \leqslant$ $0.89)$, with a median redshift of $z=0.42$. These clusters are also among the most massive known, with a median mass of $M_{500}=9 \times 10^{14} M_{\odot}$. All of these clusters have Chandra X-ray observations, and we have used these X-ray data to determine the mass of each cluster. Using these masses, we have scaled each SZ pressure profile by the mass-and-redshift-dependent normalization factor $P_{500}$ and by the overdensity radius $R_{500}$. We constrained the average pressure profile of the BOXSZ sample using a joint deprojection technique, using 13 radial bins approximately logarithmically spaced between $0.07 R_{500}$ and $3.5 R_{500}$. We note that, since the cluster virial radius is generally near $2 R_{500}$ (Umetsu et al. 2011), our deprojected pressure profiles extend beyond the virial radius, although only in two deprojection bins, each with an $\mathrm{S} / \mathrm{N} \simeq 1.5$.

The X-ray data were also used to classify the disturbed and cool-core subsamples of the BOXSZ, and we deprojected the average pressure profiles of these two subsamples into seven radial bins spanning the same radial range $\left(0.07 R_{500}\right.$ to $\left.3.5 R_{500}\right)$. We fit gNFW models to all three of these average deprojected pressure profiles, and we find that this model describes our data with a sufficient goodness of fit when $4 / 5$ of the gNFW parameters are allowed to vary. The best-fit average pressure profile of our full sample is described by the parameters $\left(C_{500}\right.$, $\left.\alpha, \beta, \gamma, P_{0}=1.18,0.86,3.67,0.67,4.29\right)$. We find a worse, although acceptable, fit quality using a $\beta$-model, but we do not explore an in-depth analysis of $\beta$-model fits due to the known shortcomings of that model (Mohr et al. 1999; Hallman et al. 2007). The gNFW fits show consistent pressure profiles regardless of cluster morphological classification outside of $\simeq 0.15 R_{500}$ given our measurement uncertainties, but inside that radius the cool-core systems show higher pressures than the disturbed systems. Due to the large parameter degeneracies in the gNFW model, our best-fit parameter values are not in general similar to those found in previous analyses (Nagai et al. 2007; Plagge et al. 2010; A10; Sun et al. 2011; P12). However, the actual profile shapes are quite similar, although our data provide hints of slightly higher pressures at both the smallest and largest radii. This agreement provides further evidence that the average cluster pressure profile is approximately universal (at least within our measurement uncertainties, which are $\simeq 10 \%-20 \%$ within $R_{500}$ ), especially given the large differences in sample masses and redshifts between the BOXSZ and these previous analyses. In addition, since many of the previous analyses relied on X-ray data, rather than SZ data, our results show the good agreement of SZ and X-ray measurements of the ICM (Plagge et al. 2010; Melin et al. 2011; Planck Collaboration 2011; Komatsu et al. 2011; Bonamente et al. 2012; P12).

Finally, we simultaneously fit for the overall mass scaling, the ensemble mean profile, and the radius-dependent intrinsic scatter of the pressure profiles using a Gaussian process model. We find that the fractional scatter is minimized at radii between $\simeq 0.2 R_{500}$ and $\simeq 0.5 R_{500}$ at values $\lesssim 20 \%$, with larger scatter at both smaller and larger radii. The best-fit mass scaling has a power-law slope of 0.49 (compared with the nominal prediction of $2 / 3$ based on self-similar hydrostatic equilibrium models), which is nearly identical to the scaling expected from the X-ray derived $Y_{X}-M$ scaling determined by M10 using an identical $X$-ray mass determination. Given our measurement uncertainties, our intrinsic scatter constraints as a function of radius are consistent with previous analyses that have largely relied on $\mathrm{X}$-ray measurements of lower mass and lower redshift clusters (A10; Sun et al. 2011; P12). This result provides additional evidence that pressure profiles are approximately universal over a wide range of masses and redshifts, with intrinsic scatter of $\simeq 20 \%-40 \%$ about the universal profile, and that SZ and X-ray measurements of these profiles are consistent with each other given current observational precision.

We acknowledge the assistance of the following: the day crew and Hilo staff of the Caltech Submillimeter Observatory, who provided invaluable assistance during data-taking for this data set; Kathy Deniston, Barbara Wertz, and Diana Bisel, who provided effective administrative support at Caltech and in Hilo; Matt Hollister and Matt Ferry, who assisted in the collection of these data; and the referee, who provided numerous useful suggestions. The Bolocam observations were supported by the Gordon and Betty Moore Foundation. J.S. was supported by a NASA Graduate Student Research Fellowship, a NASA Postdoctoral Program Fellowship, NSF/AST-0838261 and NASA/ NNX11AB07G; T.M. was supported by NASA through the Einstein Fellowship Program grant PF0-110077; N.C. was partially supported by a NASA Graduate Student Research Fellowship; A.M. was partially supported by NSF/AST-0838187; S.A., E.P., and J.A.S. were partially supported by NASA/ NNX07AH59G; K.U. acknowledges partial support from the National Science Council of Taiwan grant NSC100-2112-M001-008-MY3 and from the Academia Sinica Career Development Award. A portion of this research was carried out at the Jet Propulsion Laboratory, California Institute of Technology, under a contract with the National Aeronautics and Space Administration. This research made use of the Caltech Submillimeter Observatory, which is operated by the California Institute of Technology under cooperative agreement with the National Science Foundation (NSF/AST-0838261).

\section{REFERENCES}

Allen, S. W., Rapetti, D. A., Schmidt, R. W., et al. 2008, MNRAS, 383, 879 Arnaud, M. 2009, A\&A, 500, 103, (A10)

Arnaud, M., Pratt, G. W., Piffaretti, R., et al. 2010, A\&A, 517, A92 Bautz, M. W., Miller, E. D., Sanders, J. S., et al. 2009, PASJ, 61, 1117 Bielby, R. M., \& Shanks, T. 2007, MNRAS, 382, 1196 Böhringer, H., Pratt, G. W., Arnaud, M., et al. 2010, A\&A, 514, A32 Bonamente, M., Hasler, N., Bulbul, E., et al. 2012, NJPh, 14, 025010 Borgani, S., Murante, G., Springel, V., et al. 2004, MNRAS, 348, 1078 Cavagnolo, K. W., Donahue, M., Voit, G. M., \& Sun, M. 2009, ApJS, 182, 12 Cavaliere, A., \& Fusco-Femiano, R. 1976, A\&A, 49, 137

Cavaliere, A., \& Fusco-Femiano, R. 1978, A\&A, 70, 677

Christensen, L., Richard, J., Hjorth, J., et al. 2012, MNRAS, 427, 1953 Condon, J. J., Cotton, W. D., Greisen, E. W., et al. 1998, AJ, 115, 1963 Croston, J. H., Pratt, G. W., Böringer, H., et al. 2008, A\&A, 487, 431 Ebeling, H., Barrett, E., Donovan, D., et al. 2007, ApJL, 661, L33 Ebeling, H., Edge, A. C., \& Henry, J. P. 2001, ApJ, 533, 668 Eckert, D., Vazza, F., Ettori, S., et al. 2012, A\&A, 541, A57 Edge, A. C., Ebeling, H., Bremer, M., et al. 2003, MNRAS, 339, 913 George, M., Fabian, A., Sanders, J., et al. 2009, MNRAS, 395, 657 Glenn, J., Bock, J. J., Chattopadhyaya, G., et al. 1998, Proc. SPIE, 3357, 326 Griffin, M. J., \& Orton, G. S. 1993, Icar, 105, 537

Haig, D. J., Ade, P. A. R., Aguirre, J. E., et al. 2004, Proc. SPIE, 5498, 78 Hallman, E. J., Burns, J. O., Motl, P. M., \& Norman, M. L. 2007, ApJ, 665,911

Horner, D. J., Mushotzky, R. F., \& Scharf, C. A. 1999, ApJ, 520, 78 Itoh, N., Kohyama, Y., \& Nozawa, S. 1998, ApJ, 502, 7 
Jee, M. J., Ford, H. C., Illingworth, G. D., et al. 2007, ApJ, 661, 728

Jones, C., \& Forman, W. 1984, ApJ, 276, 38

Kaiser, N. 1986, MNRAS, 222, 323

Keisler, R., Reichardt, C. L., Aird, K. A., et al. 2011, ApJ, 743, 28K

Komatsu, R., Smith, K. M., Dunkley, J., et al. 2011, ApJS, 192, 18

Kovacs, A., Chapman, S. C., Dowell, C. D., et al. 2006, ApJ, 650, 592

Kravtsov, A. V., \& Borgani, S. 2012, ARA\&A, 50, 353

Leccardi, A., \& Molendi, S. 2008, A\&A, 486, 359

Lieu, R., Mittaz, J. P. D., \& Zhang, S.-N. 2006, ApJ, 648, 176

Mantz, A. 2009, PhD thesis, Stanford Univ.

Mantz, A., \& Allen, S. W. 2011, arXiv:1106.4052

Mantz, A., Allen, S. W., Ebeling, H., Rapetti, D., \& Drlica-Wagner, A. 2010, MNRAS, 406, 1773

Mardia, K. V. 1970, Biometrika, 57, 519

Markwardt, C. B. 2009, in ASP Conf. Ser. 411, Astronomical Data Analysis Software and Systems XVIII, ed. D. A. Bohlender, D. Durand, \& P. Dowler (San Francisco, CA: ASP), 251

Maughan, B. J., Giles, P. A., Randall, S. W., Jones, C., \& Forman, W. R. 2012, MNRAS, 421, 1583

Maughan, B. J., Jones, C., Forman, W., \& Van Speybroeck, L. 2008, ApJS, 174,117

Melin, J.-B., Bartlett, J. G., Delabrouille, J., et al. 2011, A\&A, 525, A139

Mohr, J. J., Mathiesen, B., \& Evrard, A. E. 1999, ApJ, 517, 627

Mroczkowski, T., Dicker, S., Sayers, J., et al. 2012, ApJ, 761, 47

Nagai, D., Kravtsov, A. V., \& Vikhlinin, A. 2007, ApJ, 668, 1

Navarro, J. F., Frenk, C. S., \& White, S. D. M. 1996, ApJ, 462, 563

Peterson, J. R., et al. 2001, A\&A, 365, L104

Piffaretti, R., \& Valdarnini, R. 2008, A\&A, 491, 71

Plagge, T., Benson, B. A., Ade, P. A. R., et al. 2010, ApJ, 716, 1118

Planck Collaboration 2011, A\&A, 536, A10 (early results X)

Planck Collaboration 2013, A\&A, 550, A131 (intermediate results V)

Poole, G. B., Fardal, M. A., Babul, A., et al. 2006, MNRAS, 373, 881

Postman, M., Coe, D., Benitez, N., et al. 2012, ApJS, 199, 25
Pratt, G. W., Arnaud, M., Piffaretti, R., et al. 2010, A\&A, 511, A85

Pratt, G. W., Arnaud, M., \& Pointecouteau, E. 2006, A\&A, 446, 429

Pratt, G. W., Böringer, H., Croston, J. H., et al. 2007, A\&A, 461, 71

Pratt, G. W., Croston, J. H., Arnaud, M., \& Böhringer, H. 2009, A\&A, 498, 361

Rasmussen, C. E., \& Williams, C. K. I. 2006, Gaussian Processes for Machine Learning (Cambridge, MA: MIT)

Reichardt, C. L., Shaw, L., Zahn, O., et al. 2012, ApJ, 755, 70

Reiprich, T. H., Hudson, D., Zhang, Y.-Y., et al. 2009, A\&A, 501, 899

Richard, J., Kneib, J.-P., Limousin, M., Edge, A., \& Jullo, E. 2010, MNRAS, 402, L44

Rozo, E., Bartlett, J. G., Evrard, A. E., \& Rykoff, E. S. 2012a, arXiv:1204.6305

Rozo, E., Evrard, A. E., Rykoff, E. S., \& Bartlett, J. G. 2012b, arXiv:1204.6292 Sandell, G. 1994, MNRAS, 271, 75

Santos, J. S., Tozzi, P., Rosati, P., \& Böringer, H. 2010, A\&A, 521, A64

Sayers, J., Czakon, N. G., Bridge, C., et al. 2012a, ApJL, 749, L15

Sayers, J., Czakon, N. G., \& Golwala, S. R. 2012b, ApJ, 744, 169

Sayers, J., Golwala, S. R., Ameglio, S., \& Pierpaoli, E. 2011, ApJ, 728, 39

Sayers, J., Mroczkowski, T., Czakon, N. G., et al. 2013, ApJ, 764, 152

Schmidt, R. W., Allen, S. W., \& Fabian, A. C. 2001, MNRAS, 327, 1057

Simionescu, A., Allen, S. W., Mantz, A., et al. 2011, Sci, 331, 1576

Sun, M., Sehgal, N., Voit, G. M., et al. 2011, ApJL, 727, L49

Sunyaev, R. A., \& Zel'dovich, Y. B. 1972, CoASP, 4, 173

Tran, K.-V. H., Franx, M., Illingworth, G. D., et al. 2007, ApJ, 661, 750

Umetsu, K., Broadhurst, T., Zitrin, A., Medezinski, E., \& Hsu, L.-Y. 2011, ApJ, 729,127

Vikhlinin, A., Burenin, R., Forman, W. R., et al. 2007, in Heating versus Cooling in Galaxies and Clusters of Galaxies, Eso Astrophysics Symposia, ed. H. Böhringer et al. (Berlin: Springer-Verlag), 48

Vikhlinin, A., Forman, W., \& Jones, C. 1999, ApJ, 525, 47

Vikhlinin, A., Kravtsov, A., Forman, W., et al. 2006, ApJ, 640, 691

Walker, S. A., Fabian, A. C., Sanders, J. S., \& George, M. R. 2012, MNRAS, 427, L45 